Revue des patrimoines

"Découvrir » la peinture murale au XIX ${ }^{\mathrm{e}}$ siècle : le rôle pionnier du Comité historique des Arts et Monuments dans la connaissance, la diffusion et la conservation du décor peint (1835-1852)

\title{
Élodie Jeannest
}

\section{OpenEdition}

Journals

Édition électronique

URL : http://journals.openedition.org/insitu/10832

DOI : $10.4000 /$ insitu. 10832

ISSN : 1630-7305

Éditeur

Ministère de la culture

Référence électronique

Élodie Jeannest, « « Découvrir » la peinture murale au XIXe siècle : le rôle pionnier du Comité historique des Arts et Monuments dans la connaissance, la diffusion et la conservation du décor peint

(1835-1852) », In Situ [En ligne], 22 | 2013, mis en ligne le 21 novembre 2013, consulté le 19 avril 2019 URL : http://journals.openedition.org/insitu/10832 ; DOI : 10.4000/insitu.10832

Ce document a été généré automatiquement le 19 avril 2019

\section{(†)

In Situ Revues des patrimoines est mis à disposition selon les termes de la licence Creative Commons Attribution - Pas d'Utilisation Commerciale - Pas de Modification 4.0 International. 


\title{
« Découvrir » la peinture murale au $\mathrm{XIX}^{\mathrm{e}}$ siècle : le rôle pionnier $\mathrm{du}$ Comité historique des Arts et Monuments dans la connaissance, la diffusion et la conservation du décor peint (1835-1852)
}

\author{
Élodie Jeannest
}

\section{Introduction}

1 Le rôle du Service des Monuments historiques dans la mise en place d'une politique de conservation de la peinture monumentale a été valorisé ces vingt dernières années par l'exposition ou la publication de sa collection de relevés ${ }^{1}$. Celui du Comité historique des Arts et Monuments, institué par F. Guizot en 1835, soit deux ans avant la création de la Commission des Monuments Historiques, n'en n'est pas moins important, mais restait à déterminer clairement ${ }^{2}$. Le sous-comité du Comité des travaux historiques et scientifiques qui a pour principale mission d'encourager la diffusion de documents relatifs à l'histoire des arts et de l'archéologie a en effet joué un rôle décisif dans la divulgation des connaissances dans ce domaine de recherche en pleine éclosion. À une époque où les décors peints des églises commencent à peine à être dégagés, les procès-verbaux des séances et la publication de son périodique, le Bulletin archéologique, manifestent l'intérêt, les réflexions et les actions plurielles de ce nouvel organisme. Proche du terrain, grâce à l'important réseau de correspondants qu'il a tissé, le Comité reçoit nombre de rapports et de relevés sur les peintures murales découvertes lors de prospections ou de chantiers de restauration. La première période d'activités du Comité, de 1835 à 1852, présentée dans cet article, dévoile les premiers thèmes abordés par ses membres, notamment les débats 
sur les problèmes liés au débadigeonnage, les polémiques autour des nouveaux décors ou autour de confusions iconographiques. Enfin, cette étude met en lumière l'implication active du Comité dans les publications et ses interventions auprès des autres acteurs des différentes instances publiques qui ont souvent été suivies, complétées ou concurrencées par l'action de la Commission des Monuments historiques, notamment dans la production et l'utilisation des relevés.

\section{Un cadre favorable à la réception d'informations sur le décor peint : la genèse du Comité des Arts et Monuments}

\section{Les étapes de création officielle et les fonctions principales du Comité des Arts et Monuments}

2 Institué sur l'initiative du ministre de l'Instruction publique F. Guizot, féru d'histoire et d'archéologie, dans un contexte d'engouement pour l'art médiéval et propice à la recherche archéologique, le Comité historique des Arts et Monuments est promis à une belle place dans l'histoire de la diffusion de l'érudition. Il se fond, à l'origine, dans l'une des cinq entités historiques du Comité des Travaux historiques et scientifiques voulu par la Monarchie de Juillet et créé en 1834 pour devenir l'organe officiel et centralisé de diffusion de tous les documents intéressant l'histoire de France ${ }^{3}$. Un arrêté du 10 janvier 1835 institue plus précisément le " second Comité », officiellement nommé « Comité pour l'histoire morale et intellectuelle de la France ». La nomination de huit membres dont la majorité est reconnue pour sa connaissance du domaine archéologique oriente clairement cette entité vers le domaine des monuments et des arts. Durant l'année 1835, A. Leprévost, V. Hugo, L. Vitet et P. Mérimée, C. Lenormant et A. Lenoir y composent un premier sous-comité des Arts ${ }^{4}$. Le 18 juillet 1837, N. de Salvandy, ministre de l'Instruction publique, érige officiellement le sous-comité des Arts en Comité historique des Arts et Monuments 5 . Il devient, de manière visible, l'un des cinq comités réorganisés. Preuve de son bon fonctionnement, ce comité reste indépendant, lorsqu'en 1840 les quatre autres fusionnent pour devenir le Comité des Monuments écrits. Ce Comité des Arts et Monuments ${ }^{6}$, placé sous la tutelle de l'Académie des Beaux-Arts, incarne rapidement la volonté interventionniste de l'État par sa centralisation des informations dans le domaine archéologique, sujet récurrent de confrontation entre l'administration centrale et les municipalités. Le Comité, s'il est tout d'abord critique envers les acteurs de la restauration, membres du clergé, des sociétés savantes et des différentes commissions départementales, devient toutefois progressivement la tribune des contestations provinciales contre les représentants de l'État. Lorsqu'en 1848, une réforme ajoute la responsabilité des cultes au ministère de l'Instruction publique, les membres les plus revendicatifs, A. Didron, V. Hugo et C. de Montalembert sont écartés du Comité devenu "Commission des Arts et Monuments diocésains ». Après le coup d'État du 2 décembre 1851, Louis Napoléon Bonaparte lui impose d'abandonner définitivement les polémiques pour ne plus se consacrer qu'aux seuls enjeux scientifiques. Ce muselage précède une réorganisation générale du Comité en même temps qu'un contrôle amplifié des sociétés savantes. L'arrêté du 14 septembre 1852 émis par H. Fourtoul ouvre une nouvelle et longue période pendant laquelle le Comité des Monuments écrits et le Comité des Arts et Monuments sont regroupés en un seul Comité. Intitulé désormais «Comité de la Langue, 
de l'Histoire et des Arts de la France ", il est divisé en trois sections : philologie, histoire et archéologie. Sa priorité, bien que ses missions demeurent à priori identiques, consiste en la valorisation renforcée de l'histoire de la langue française et de l'histoire de la Nation'.

Créé pour « Mettre en lumière les monuments inédits de l'histoire de France », le Comité développe, sous la direction du ministère de l'Instruction publique, un véritable processus de divulgation des connaissances dans le domaine de l'archéologie médiévale ${ }^{8}$. Véritable relais entre les différents acteurs du patrimoine, il devient un puissant promoteur de connaissances, grâce à son intense politique de publications ${ }^{9}$. Dès les premières séances de travail, les membres du Comité organisent des méthodes précises d'inventaire pour donner des informations sur l'ensemble des monuments. Il s'efforce également de permettre aux acteurs du patrimoine, érudits, restaurateurs et architectes chargés des restaurations de s'approprier des procédés de travail généralisés en leur proposant des Instructions, publiées tout le long de cette première période. Il outrepasse même ce rôle de conseiller et de représentant en s'octroyant la responsabilité de conserver les monuments historiques. Cette position ouvertement assumée ne va pas sans se heurter dès l'origine aux prérogatives d'autres institutions étatiques officiellement responsables de cette fonction $^{10}$. Grâce à son rôle d'intermédiaire, le Comité centralise les énergies et les enthousiasmes des sociétés savantes dont le régime s'est libéralisé en avril 1834. Il permet également de servir de relais entre les différents autres acteurs de la restauration patrimoniale qui sont dispersés dans les différents ministères. Ce partage des informations est également possible grâce aux cumuls des fonctions de certains correspondants et à une majorité des membres du Comité ${ }^{11}$. C'est le cas de P. Mérimée, nommé inspecteur des Monuments historiques en 1834 ou encore de J. Schmit, attaché au ministère de la Justice et des Cultes ${ }^{12}$.

\section{Ses moyens mis en œuvres : un réseau de correspondants dynamiques}

4 L'action de rassembler des informations sur tous les monuments de France, et, de fait, sur les peintures murales ornant ces édifices, n'aurait pu voir le jour sans l'aide d'hommes de terrain, au plus proche du « document archéologique ». Ces hommes étaient d'ailleurs le plus souvent déjà investis d'une fonction dans le domaine archéologique provincial. Leur nomination est cependant tributaire d'un certain nombre de compétences et de devoirs exigés par leurs missions, et précisément des connaissances préalables en archéologie et une habileté de dessinateur ${ }^{13}$. Cette dernière qualité a joué en faveur de la diffusion de la connaissance sur la peinture murale. L'idée qu'« un dessin, quelque mauvais qu'il soit, en dit plus sur l'âge, le style et l'importance d'un monument d'architecture, de sculpture et de peinture, que des pages entières de descriptions ${ }^{14}$, ainsi que l'envoi de documents graphiques exigés au même titre que le rapport écrit pour chaque sujet énoncé, ont permis au Comité de se constituer un fonds de relevés en couleur, fondamental pour la publication future de planches illustrées. Si les premières informations données sur la peinture murale en France sont dépendantes du talent de dessinateur de leurs rapporteurs, elles sont aussi soumises aux nominations géographiques des correspondants. En début de l'année 1842, un membre du Comité indique que plus d'un quart des départements n'est pas encore représenté par un correspondant: leurs patrimoines archéologiques sont donc méconnus, voire inconnus pour le Comité ${ }^{15}$. Enfin il faut compter sur l'intérêt particulier de certains membres et correspondants pour la 
peinture murale qui a permis au Comité de recevoir un grand nombre de rapports, notices et relevés sur le sujet.

\section{Des rapports et des dessins : la prise de conscience de la richesse du sujet}

$5 \quad$ Le premier sujet ayant intéressé le Comité dans le domaine du décor peint concerne l'acte du grattage. Les voix des membres du Comité, et notamment celle tonitruante de V. Hugo, fait écho aux circulaires déjà envoyées en ce sens par le ministère de la Justice et des Cultes ${ }^{16}$. Lors de la séance du 29 mars 1835 rapportant les dommages réalisés à l'Hôtel de Ville de Paris et l'église Saint-Paul, et les menaces qui pèsent sur la cathédrale NotreDame de Paris, l'écrivain réclame « qu'on s'oppose par tous les moyens à cette fureur de gratter et que les circulaires du Comité se prononcent formellement à cet égard. $»^{17}$. Cette problématique du grattage, de même que celles du débadigeonnage et du badigeonnage, se rencontre régulièrement dans les procès-verbaux divulgués dans la revue officielle du Comité.

6 Les premiers décors peints mentionnés devant le Comité sont ceux de Saint-Savin-surGartempe et de Saint-Jean de Poitiers. P. Mérimée avait déjà fait état de leur condition déplorable auprès du ministre de l'Instruction publique en 1835 et rédigé une première notice en $1836^{18}$. Lors de la séance du 31 janvier 1838, l'inspecteur des Monuments historiques invite alors les membres du Comité à agir en faveur de ce patrimoine en péril 19. Parmi les membres du Comité, A. de Bastard joue aussi un rôle significatif pour la diffusion d'informations sur la peinture murale ${ }^{20}$. Après avoir évoqué en séance le travail de relevés qu'il a fait exécuter sur les peintures de Saint-Savin-sur-Gartempe, il se penche ensuite, à la demande du ministre de la Guerre, sur les problèmes de conservation des peintures de l'abbaye de Saint-Jean-des-Vignes à Soissons. Il retourne au ministre des préconisations simples mais efficaces: "À la recommandation du Comité des Arts et Monuments, des peintures à fresque qui ornent le dortoir de Saint-Jean-des-Vignes, à Soissons, lequel appartient à la Guerre, ont été préservées, par des volets, de la poussière et de la dégradation ${ }^{21}$. On peut également noter les nombreux envois de C. Texier, membre du Comité depuis 1837, grâce auquel un grand nombre de peintures murales sont signalées au Comité22. Ces signalements correspondent véritablement à une prise de conscience d'une lacune dans ce domaine de l'histoire de l'archéologie des peintures murales: "Dans une excursion récente, j'ai recherché avec soin les fragments d'anciennes peintures murales, conservées dans nos vieux monuments. Mes explorations ont été peu fécondes en résultats importants. Mais, dans un champ si pauvre et si peu exploré les moindres renseignements peuvent avoir de l'intérêt $»^{23}$. Ainsi, les premières attentions portées sur le décor peint proviennent de membres actifs du Comité.

7 La diffusion de la connaissance dans ce domaine n'aurait également jamais pu avoir lieu sans la présence de correspondants qui présentaient un intérêt particulier envers les décors polychromes. Le projet de dresser une statistique monumentale mettant en avant chaque monument devient l'occasion de découvrir à l'intérieur des édifices leurs décors peints. Ainsi peut-on noter les noms de S. Boisserée, membre des Académies des Sciences et Arts de la Bavière et correspondant de l'Institut de France, de son frère Melchior, ou encore du correspondant belge Jénicot ${ }^{24}$. Au gré des découvertes, ce n'est pas seulement la peinture murale médiévale qui est renseignée mais un répertoire historique beaucoup plus vaste, de l'Antiquité au XVIII ${ }^{\mathrm{e}}$ siècle, de la Creuse jusqu'à Westminster ${ }^{25}$. Tous les 
édifices sont bons à découvrir : l'édifice antique déblayé laisse apparaitre des peintures murales, à l'instar d'un édifice à Villars ou à Saint-Trophime d'Arles. Parfois le décor primitif est dégagé de plusieurs couches successives de décors peints qu'il a fallu faire disparaitre définitivement pour l'atteindre, comme le décor d'amphitrite de la chapelle Sainte-Agathe de Langon $^{26}$ (fig. $\left.\mathbf{n}^{\circ} \mathbf{1}\right)$ (fig. $\mathbf{n}^{\circ} \mathbf{2}$ ). Les décors postérieurs à l'époque médiévale sont également l'objet d'études et d'un suivi consciencieux ${ }^{27}$. Face à la menace qui pèse dès 1845 contre les célèbres fresques de Romanelli ornant le Palais Mazarin, sujet d'un projet de destruction, A. de Laborde est chargé de rédiger une note ${ }^{28}$. Les découvertes et interrogations qui se jouent aux frontières belges intéressent aussi grandement le Comité. L'action dynamique et curieuse du secrétaire de la Société d'émulation de Liège dans la mise en place d'une politique de conservation des peintures de l'église Saint-Jacques se suit sur plusieurs années, de même que le sort des peintures de l'église de Notre-Dame de Tournai ${ }^{29}$. Les correspondants divulguent également des informations sur les peintures de Bethléem ou sur de nombreux décors italiens, comme celui de la vie de saint François d'Assise de Santa Croce ${ }^{30}$. L'annonce et l'envoi des publications entreprises à l'échelle régionale par les correspondants et les commissions archéologiques sont également autant de moyens de faire connaitre les découvertes récentes ou les travaux scientifiques entrepris sur les décors. Exemples parmi d'autres, F. Mandet rend compte dès 1840 de la description détaillée de la Danse des Morts de l'église de La Chaise Dieu et d'une peinture malheureusement badigeonnée à Saint-Michell'Aiguilhe. A. du Mège, correspondant à Toulouse, apporte, quant à lui, des informations sur la dépose de peintures dans le Languedoc ${ }^{31}$. Les membres du Comité prennent également conscience que les édifices civils, notamment les châteaux, sont dotés de décors peints, ceux des châteaux de Capestang ou de Cindré sont ainsi révélés ${ }^{32}$. 
Figure 1

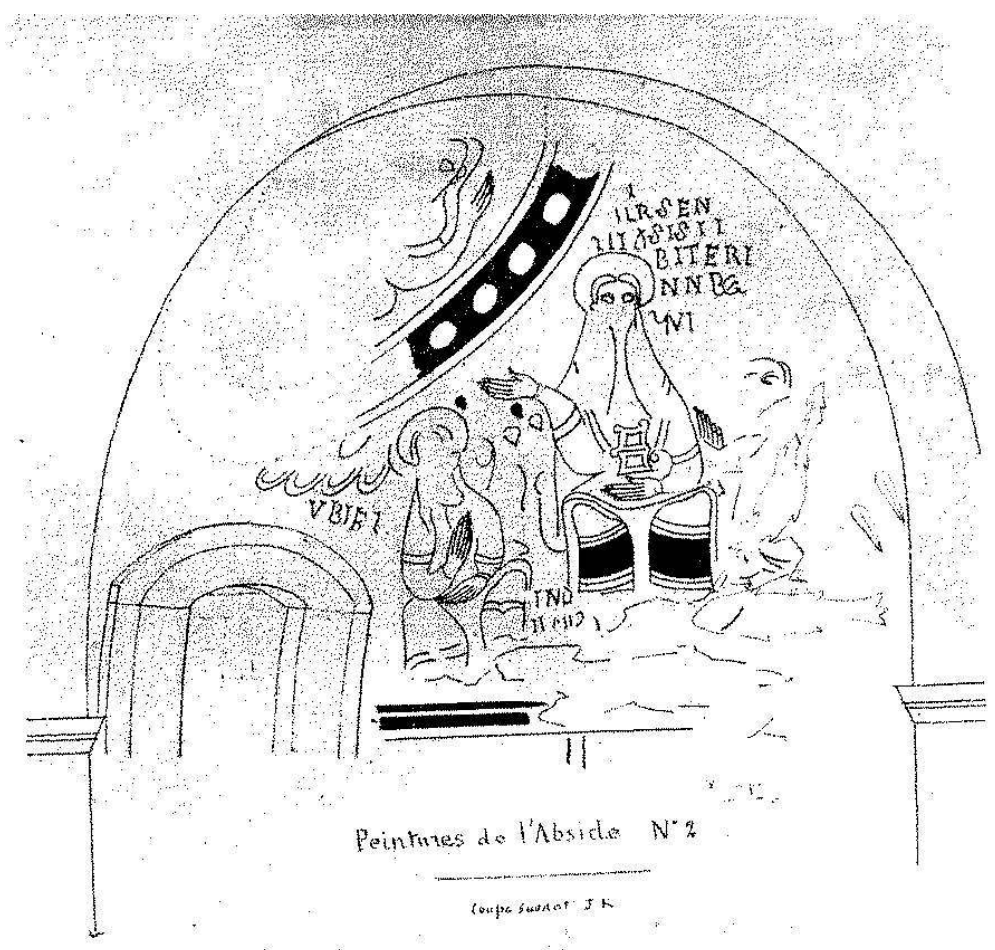

Détails d'ornements des peintures de la voûte de la chapelle Sainte-Agathe de Langon. Relevé de Langlois, $1839, n^{\circ} 01271$

Phot. Médiathèque de l'architecture et du Patrimoine. (c) Ministère de la Culture, Médiathèque de l'architecture et du patrimoine. 


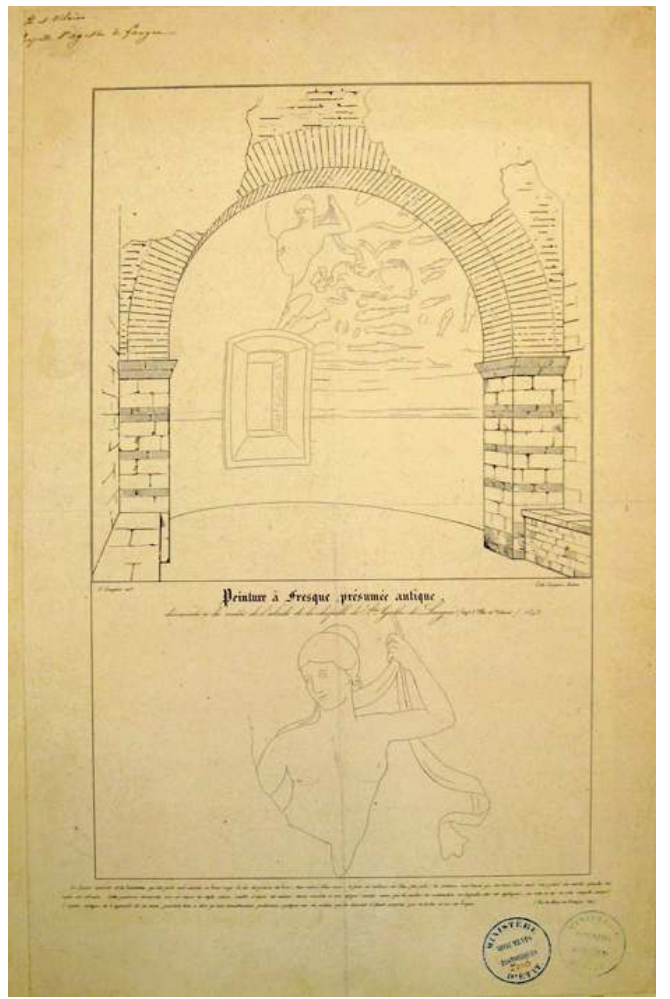

Décor primitif représentant une amphitrite de la chapelle Sainte-Agathe de Langon. Gravure d'après un relevé de Langlois, $n^{\circ} 05010$

Phot. Médiathèque de l'architecture et du Patrimoine. (c) Ministère de la Culture, Médiathèque de l'architecture et du patrimoine

L'obligation d'envoi de dessins en complément des rapports a offert aux correspondants l'opportunité de faire valoir leur talent dans la restitution des peintures murales. Les mentions de ces envois sont si importantes dans les archives du Comité qu'il est impossible d'en faire une liste exhaustive (fig. $\mathbf{n}^{\circ} \mathbf{3}$ ). Dans le cadre du projet d'élaboration de la statistique monumentale nationale, L. Vitet propose, dès le 18 janvier 1835 , de «former une collection de dessins que l'on publierait ». Cette idée est encore énoncée en séance du 23 novembre 1840, ciblant même plus précisément «les monuments de la peinture sur bois, murs ou sur verre ${ }^{33} »$. Le rapporteur avance également l'hypothèse selon laquelle l'intervention d'un dessinateur et son interprétation pourrait être une étape capitale dans la connaissance de l'œuvre: «Il faudrait les dessiner et pour ainsi dire, les rendre lisible ». Ces dessins permettent par ailleurs de conserver un témoignage de peintures promises à la destruction, comme c'est le cas des peintures du château de Cindré $^{34}$. Cette initiative du relevé pris en anticipation de la destruction se développe même, à l'instigation notamment de l'inspecteur des Monuments historiques, comme moyen de substitution aux opérations de restauration ${ }^{35}$. La découverte de la peinture murale en France à la fin du premier tiers du XIX siècle repose donc sur une action de terrain de correspondants, relayée par un organisme centralisateur aux membres puissants et aux réseaux étendus. L'ouvrage de T.B. Émeric-David, intitulé l'Histoire de la peinture du Moyen Age depuis Constantin jusqu'au XII siècle et publié dès 1811, manifeste précocement l'existence de la peinture murale médiévale à une période où elle demeure encore bien méconnue, le mécanisme de transmission et de publication mis en place par 
le Comité des Arts et Monuments a joué cependant un rôle capital de valorisation du décor peint, car pour la première fois officiellement issu d'une administration $^{36}$. Le Comité devient donc le réceptacle de l'information sur les découvertes et les dangers encourus par la peinture murale. Il s'efforce de répondre aux diverses demandes de ses correspondants en créant des groupes de travail, un périodique qui divulgue ses positions et en encourageant les publications.

Figure 3

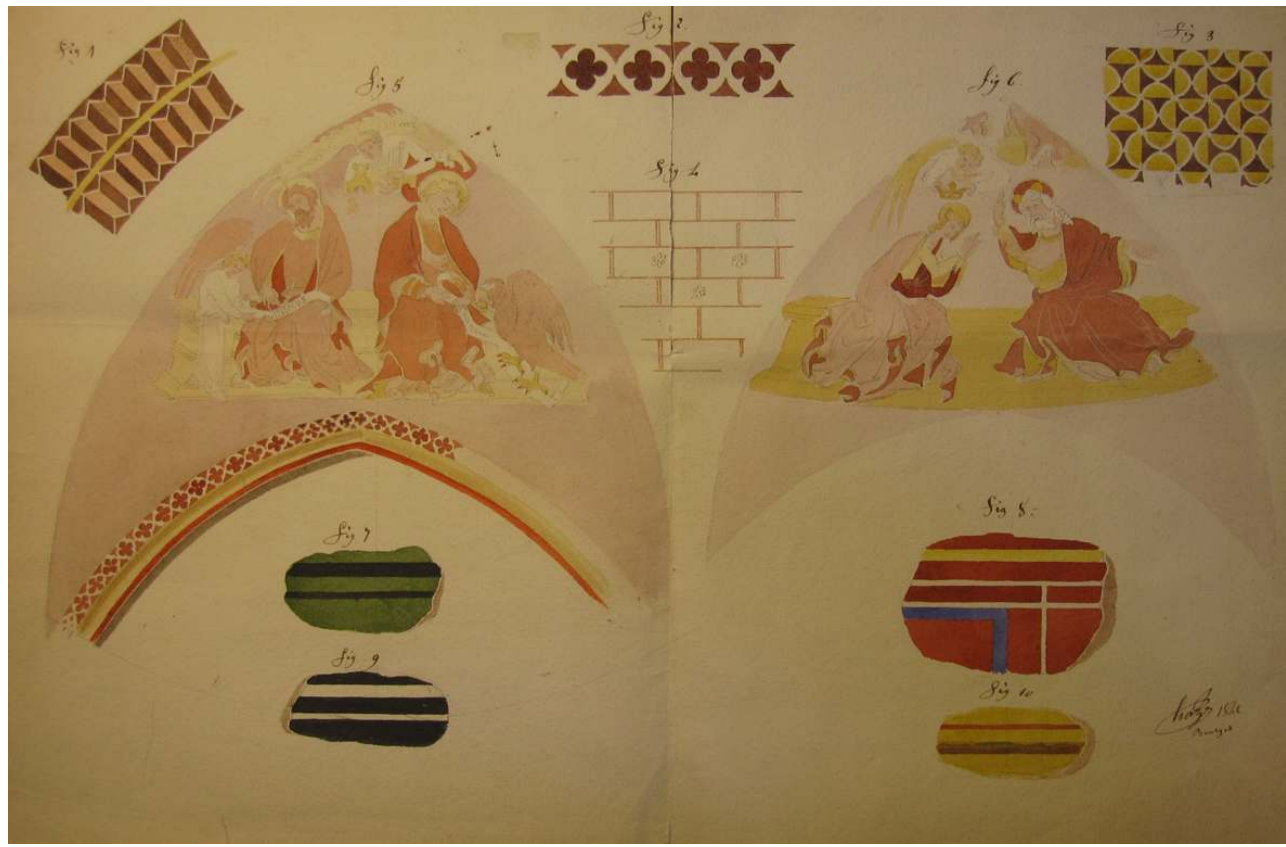

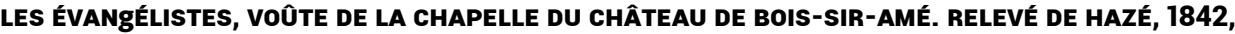
F17/2810.

Phot. Archives Nationales, Élodie Jeannest, 2012. @ Ministère de la Culture, Archives Nationales.

\section{La naissance d'une réflexion sur la valorisation et la préservation de ce patrimoine en danger}

\section{La constitution de groupes de travail}

9 Pour répondre aux questions particulières posées par ses correspondants, le Comité constitue des groupes de travail qui rassemblent un certain nombre de membres dont la connaissance du sujet et le réseau d'influence permettent d'avancer des solutions. Non seulement le Comité forme une commission pour réfléchir spécifiquement à la restauration de la cathédrale Notre-Dame de Paris, mais il invite aussi des adhérents reconnus pour leur engagement en faveur des monuments à en composer une autre pour travailler sur le devenir des fresques de Saint-Savin-sur-Gartempe ${ }^{37}$. Une troisième est, quant à elle, chargée de trouver les meilleurs moyens de débadigeonner l'intérieur des monuments ${ }^{38}$. Pour compléter ses réflexions, le Comité passe commande aux correspondants, les encourage à approfondir leurs recherches, les interroge : c'est le cas notamment pour les peintures de Saint-Gilles de Montoire. Après avoir reçu un premier rapport de son correspondant à Vendôme, il l'invite à renvoyer d'autres informations sur 
les peintures et de les compléter par des dessins ${ }^{39}$. Un réel échange se tisse entre les acteurs de terrain et les têtes pensantes qui siègent au Comité, tout au profit de la connaissance de la peinture murale.

\section{Le Bulletin archéologique : une tribune publique de diffusion} «les rapports les plus remarquables». La constitution d'archives pour y déposer l'ensemble de la correspondance et les minutes des travaux commandés par le ministère paraît également indispensable ${ }^{40}$. Le 26 février 1839, un arrêté autorise le Comité à publier une fois par mois un Bulletin archéologique reprenant les procès verbaux des séances tenues tous les 15 jours au ministère de l'Instruction publique, ainsi que les extraits les plus intéressants de sa pléthorique correspondance ${ }^{41}$. Le premier volume apparait en 1843 et porte sur les années de 1838 à $1840^{42}$. < http://gallica.bnf.fr/ ark:/12148/bpt6k208417t.image >. Dès 1844, le succès des envois des correspondants est tel que, pour ne pas les vexer, le Comité est obligé de préciser que tous les documents envoyés ne peuvent être publiés et qu'ils seront classés "aux archives où ils seront consultés en temps utile $»^{43}$. Le Comité qui ne dispose pas de véritables moyens d'actions directes sur les monuments a donc compris en cette période de développement de la presse, la nécessité et la force de ce moyen de communication à vaste spectre. Pour contrer les actes les plus malhabiles ou malveillants qui lui sont rapportés, il comprend que la dénonciation publique est un des premiers moyens pour faire réagir les instances publiques responsables. Il l'affirme clairement lors du scandale qui éclabousse le dégagement des peintures murales de la cathédrale Saint-Julien du Mans ${ }^{44}$. Il s'en sert également dès 1842 pour alerter du sort qui menace les peintures murales de l'abbaye de Charlieu. Cette dénonciation publique a d'ailleurs indubitablement contribué à la mobilisation en leur faveur de la Commission des Monuments historiques et de P. Mérimée en personne deux ans plus tard ${ }^{45}$.

\section{L'apparition de doctrines officielles}

11 À la fois dénonciateur et instructeur, Le Bulletin archéologique permet également aux membres du Comité de présenter clairement leurs doctrines dans le domaine du décor peint.

\section{Guerre aux badigeonneurs - guerre aux débadigeonneurs !}

Le Bulletin archéologique offre en effet l'opportunité aux membres de se révolter publiquement contre les actions de grattages, badigeonnages ou débadigeonnages menées par « des espèces de Normands dévastateurs $»^{46}$. Ainsi le procès verbal de la séance du 14 février 1839 rappelle que « Messieurs Vitet, Lenormant et Mérimée, de Montalembert, $\mathrm{Du}$ Sommerard, Bottée de Toulmont se prononcent énergiquement contre le badigeonnage sale et gluant dont on travestit nos monuments religieux et dont on empâte les fines sculptures ». M. Schmit relève même l'impuissance des pouvoirs en place contre ce vecteur de dégradation en prenant pour exemple le cas d'interventions de badigeonnage faites à l'encontre des peintures de Saint-Quentin de Retz, près d'Aigueperse : «la loi est muette et impuissante; l'instruction archéologique propagée par le Comité est la mesure la plus efficace contre les dégradations et destructions 
monumentales. $\aleph^{47}$ Le scandale révélé par Lassus au sujet d'actions dirigées contre la cathédrale de Chartres est aussi révélateur des actes outrageant le décor peint dénoncés par une prolifique correspondance ${ }^{48}$. Alors même que le Comité concentre une grande partie de ses efforts à chercher les moyens les plus justes de dégager les couleurs des cathédrales, d'autres responsables administratifs s'attachent à les détruire ou à les couvrir. Le cas des peintures d'Aire-sur-la-Lys et de la cathédrale du Mans illustrent parfaitement ce problème des décors dégagés de leurs badigeons (fig. $\left.\mathbf{n}^{\circ} \mathbf{4}\right)$. Les peintures découvertes par l'architecte en charge de la restauration de l'édifice sont extrêmement abîmées, soit en raison de la méthode utilisée pour les dégager, soit en raison du mauvais état de l'édifice. Ces décors anciens et dégradés déplaisent à la population locale qui souhaite les ensevelir à nouveau au profit d'un nouveau décor plus au goût du jour. Le Comité met alors en place une procédure de conservation conjointe avec les instances responsables, tout en prônant une intervention minimaliste ${ }^{49}$. En parallèle de ces récriminations, une requête lancée par le responsable de la restauration du décor mural de Saint-Jacques de Liège offre au Comité la possibilité de mener une réflexion sur le procédé le moins nocif pour le décor peint en termes de débadigeonnage. Pour ce faire, il s'appuie sur un grand nombre de témoignages rassemblés et publiés au fur et à mesure sur les procédés expérimentés ${ }^{50}$. En 1840 le ministre de l'Instruction publique invite même l'Académie des Sciences à s'interroger sur les moyens de débadigeonner les peintures sans les altérer ${ }^{51}$.

Figure 4

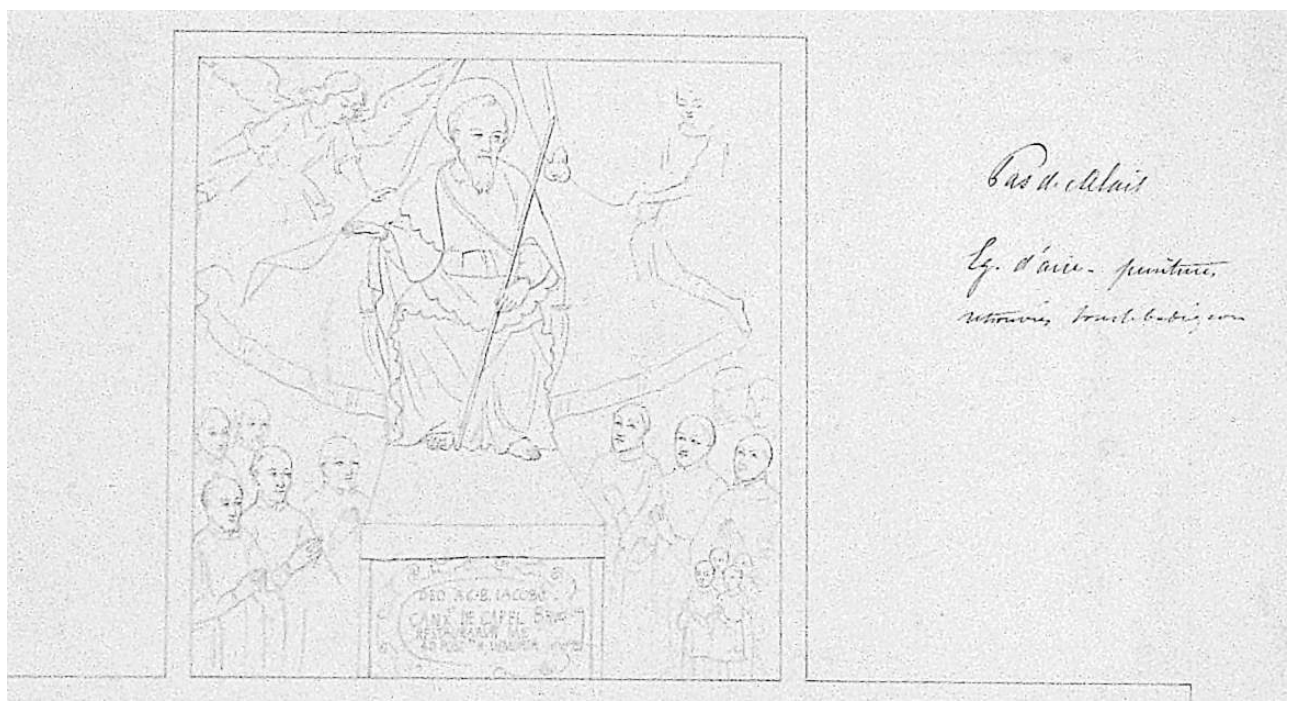

Détail d'une scène légendaire de la vie de saint Jacques de Compostelle, église Saint-Pierre d'Aire-sur-laLys. Relevé de Boileau, 1842, n02205.

Phot. Médiathèque de l'architecture et du patrimoine, Élodie Jeannest, 2012. ( ) Ministère de la Culture, Médiathèque de l'architecture et du patrimoine.

\section{La place de la peinture murale dans les nouveaux décors}

Les nombreux débats sur la restauration des édifices religieux et sur le problème de dégradation des peintures une fois dégagées, donnent l'occasion aux membres du Comité d'exprimer leur opinion sur le sujet de la reprise des décors intérieurs, soit par restauration, soit par création de nouveaux décors. L'utilisation de la peinture murale dans les nouveaux projets de décoration est d'ailleurs largement préconisée, soutenue par 
les quelques progrès réalisés dans la connaissance de la technique, qui prônent l'utilisation de la peinture à fresque ${ }^{52}$. Une séance de 1846 communiquée par le Bulletin archéologique offre l'occasion à chacun des membres du Comité d'avancer son point de vue sur la place de l'art contemporain dans les édifices religieux. I. Taylor se fait le porteparole de l'idée partagée par la majorité que la peinture murale doit être préférée à la peinture sur toile dans la décoration intérieure des édifices : « Les tableaux vont mal dans les églises, où ils brisent les lignes de l'architecture et détruisent l'effet perspectif des monuments. On dépense beaucoup d'argent pour acheter des tableaux religieux, il vaudrait mieux dépenser cet argent à commander des peintures murales ${ }^{53}$.» Cependant certains membres formulent leur inquiétude face aux nouveaux décors qui entravent l'harmonie générale d'un édifice, jugeant que «la peinture religieuse qui se fait en ce moment est mauvaise ; si l'art ne devait pas s'améliorer, il faudrait préférer les tableaux aux peintures murales car un tableau a l'immense avantage de pouvoir s'enlever, se mettre au grenier ou se brûler, tandis qu'une peinture sur mur reste longtemps en place et déshonore pour longtemps, pour toujours, un monument ${ }^{54}$ ». Ils distinguent alors les nouveaux décors programmés dans les édifices modernes et ceux se substituant à d'anciens sur des édifices médiévaux en cours de restauration. Contre les difficultés à peindre des décors muraux, A. Didron dénonce même un manque de connaissance dans le domaine de l'iconographie et de la technique, selon une théorie de la primauté de la science archéologique sur le talent artistique qu'il défend pendant de longues années ${ }^{55}$. En réalité, le Bulletin archéologique laisse transparaître une réelle méfiance généralisée ${ }^{56}$. Ce discours et cette invitation à la prudence dans le choix d'exécuter des peintures murales est d'autant plus crucial qu'il répond à la réalité de nombreux chantiers dont les correspondants les informent ${ }^{57}: 200000 \mathrm{~F}$ ont été ainsi mobilisés en 1846 pour la réalisation de nouveaux décors peints dans les édifices parisiens. Soucieux de la cohérence des bâtiments, ils demandent des informations à leurs correspondants sur le terrain. C'est notamment le cas pour l'immense chantier mené à la cathédrale NotreDame du Puy-en-Velay ${ }^{58}$. Ce dernier a d'ailleurs permis d'expérimenter le projet d'utiliser des relevés réalisés avant la destruction des peintures murales comme modèles pour une restitution à l'identique et " avec une consciencieuse fidélité » du décor ${ }^{59}$. À son manque d'enthousiasme envers la production de peinture contemporaine, s'ajoute une opposition assumée contre les interventions directes sur les peintures en matière de restauration.

\section{Une restauration minimaliste}

Les débats et les conseils demandés à propos de la restauration de l'abbaye de Saint-Jeandes-Vignes sont l'occasion pour le Comité d'annoncer précocement sa position en matière de restauration : « Consolider d'abord et avant tout ; puis restaurer, si l'on peut, telle est la doctrine du Comité des arts, telles sont surtout les instructions du gouvernement ${ }^{60} »$. La position du Comité en matière de restauration de peintures murales entre, de fait, en parfaite cohérence avec celle qu'il tient pour toute restauration d'édifice : il s'indigne des restaurations sauvages faites dans l'ignorance et l'irrespect du décor peint ${ }^{61}$. Sa doctrine est celle de ne pas toucher au décor, excepté pour le débadigeonner, au pire le consolider ${ }^{62}$. La seule intervention encouragée, et encore en toute connaissance de cause, avec l'assistance de personnes qualifiées, est celle de débadigeonner les peintures, grâce à la méthode prescrite par le Comité, sans intervention directe ultérieure ${ }^{63}$. Ce sont bien les directives données aux représentants des chantiers de Tournai et de Liège, puis du Mans. 
Grâce à sa composition, la création d'une tribune officielle et des doctrines clairement exposées, le Comité joue un rôle bien connu de nos contemporains de Lobbying. C'est tout autant par sa force de persuasion que par sa fonction première d'éditeur que le Comité mène efficacement des actions de valorisation de la peinture murale.

\section{Agir en faveur de la peinture murale : la publication en faveur de la diffusion des connaissances}

\section{La naissance des publications}

16 Le rôle principal du Comité est celui de diffuser les connaissances par le biais de diverses publications. Dès 1835 il instaure plusieurs types d'ouvrages. Au côté des Statistiques monumentales, les Instructions sont également publiées, fournissant des principes archéologiques pour chaque typologie de bâtiment ${ }^{64}$. Le Comité s'exerce aussi précocement à un autre type d'ouvrage novateur : la monographie d'édifice. En dépit de trois grands projets ayant pour sujet les cathédrales de Noyon, Chartres et de la SainteChapelle qui monopolisent attentions et subsides, un quatrième projet, à la limite de la monographie, est lancé par le Comité. Il s'agit d'un ouvrage sur la peinture murale qui offre l'occasion d'exploiter les nouvelles technologies utilisées par le monde de l'imprimerie pour restituer la couleur ${ }^{65}$. En mentionnant pour la première fois en séance les peintures de Saint-Savin-sur-Gartempe et la nécessité de les faire dessiner, Mérimée ouvre également la voie de leur publication. Il a juste été précédé par l'action déjà citée plus haut de A. de Bastard sur les peintures de Saint-Jean de Poitiers et de Saint-Savin. C'est sans doute en prenant connaissance de cette initiative que germe l'idée d'une publication ${ }^{66}$. Le 16 mai 1838, A. de Bastard renouvelle la proposition d'une publication sur les peintures murales en France qui n'a cependant pas d'effet immédiat ${ }^{67}$. L'ajournement du projet doit peut-être se comprendre comme l'ignorance encore, en cette fin des années 1830, de l'importance quantitative de ce type de témoignages de l'art médiéval. Les premières mentions des peintures murales soulignent toute la rareté ou l'unicité des décors découverts, tant la croyance est encore forte à cette période de la disparition massive de ces décors. Face à cette notion de rareté, on peut comprendre l'effet que produisit, sur les passionnés d'art médiéval, l'impressionnante voûte ornée de Saint-Savin-sur-Gartempe et leur vif désir de la faire connaitre par le biais d'une publication ${ }^{68}$. Ce projet semble d'autant plus nécessaire qu'il n'apparaît encore à cette époque aucun moyen d'endiguer le processus de dégradation des peintures murales, faute de connaissance suffisante dans le domaine de la restauration ${ }^{69}$. L'ouvrage Notice sur les peintures murales de l'église de Saint-Savin ne peut voir le jour qu'en 1845 grâce à l'action conjointe et fort intelligente du Comité et de la Commission des Monuments historiques ${ }^{70}$. Cependant, tel qu'il nous est aujourd'hui présenté, il ne témoigne pas de la complexité de sa réalisation, notamment dans le choix des décors à publier. Si les peintures murales de Saint-Savin-sur-Gartempe sont considérées comme les vedettes à mettre impérativement en valeur dans cette publication, l'idée de départ est en réalité une publication générale sur la peinture murale française $\mathrm{i}^{71}$. C'est l'interprétation que l'on peut donner de la volonté systématique d'y intégrer les décors peints les plus importants mentionnés les uns après les autres en séance. Le décor du Baptistère Saint-Jean de Poitiers qui avait été signalé au même titre que celui de l'abbaye poitevine, est ainsi inclus pour un temps dans le projet. Les chromolithographies exécutées selon les relevés entrepris par G. Séguin au 
moment de sa campagne de relevés à Saint-Savin sont finalement retirées en 1844 (fig. $\mathbf{n}^{\circ}$ 5) ${ }^{72}$. Après l'envoi de l'important travail de G. Launay sur les fresques de la chapelle de Saint-Gilles de Montoire, A. de Laborde argue encore que « les peintures murales ne sont pas assez communes en France pour qu'on ne publie ou qu'on ne signale toutes celles que nous possédons " et propose qu'elles soient intégrées à la publication ${ }^{73}$. Les dessins envoyés par ce correspondant sont finalement relégués au profit de relevés exécutés en 1841 par J.-B. Jorand ${ }^{74}$. Les peintures murales de la crypte de la cathédrale de Limoges, dont C. Texier envoie le dessin, sont également proposées à la publication ${ }^{75}$. En 1844, l'ouvrage risque même de prendre une toute autre tournure lorsqu'il est question d'y intégrer une note sur les émaux « représentant les apôtres qui décorent aujourd'hui la chapelle de la Vierge dans Saint-Pierre de Chartres ${ }^{76}$ ». Cette prise de conscience de l'importance du décor mural en France et à l'étranger ${ }^{77}$ et la multiplication des propositions d'intégration d'autres décors ont rendu finalement impossible ce projet de compilation de toutes les peintures murales médiévales françaises découvertes et conservées. Face à la multitude des envois de rapports et de dessins, le Comité soutient une nouvelle fois l'idée d'un ouvrage général sur les peintures murales françaises et renseignement est pris du coût d'une telle entreprise en 1846. Les techniques de publication en couleurs des planches de relevés posent cependant un réel problème financier qui écarte ce projet ${ }^{78}$, remplacé par la publication de nombreuses études à l'échelle régionale: les peintures murales de Montoire relevées par J.-B. Jorand sont finalement publiées en 1850 par la Société archéologique et historique de l'Orléanais ${ }^{79}$. Enfin, il est acquis que le décor peint, au même titre que le décor sculpté, doit faire partie intégrante des publications relevant de la description des édifices. Cette idée est en effet avancée dès 1840 pour l'étude commandée par le Comité sur le Palais des Papes d'Avignon, en envisageant même une campagne de relevés ${ }^{80}$. C'est le cas également pour la Statistique monumentale de Paris pour laquelle un artiste soumet au Comité des relevés de peintures ornant la cathédrale Notre-Dame de Paris ou encore pour la monographie projetée sur la Cathédrale de Chartres ${ }^{81}$. 


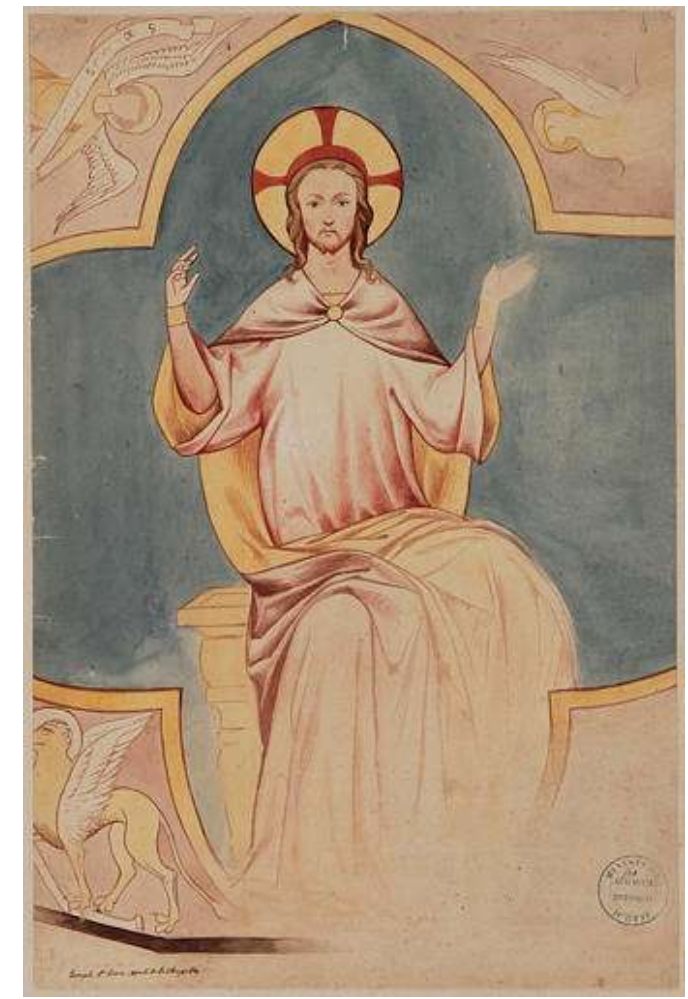

Christ en majesté, Baptistère Saint-Jean de Poitiers. Relevé de G. Séguin, 1840, n05913.

(c) Ministère de la Culture, Médiathèque de l'architecture et du patrimoine.

\section{Les progrès rencontrés dans le domaine de l'iconographie}

17 Le Comité a d'ailleurs fait preuve dès sa création d'un réel intérêt pour les avancées dans le domaine naissant de l'iconographie ${ }^{82}$. Le chapitre "Iconographie " intégré dans le Bulletin archéologique, les nombreuses pages de descriptions et l'archivage des dessins ont engendré un grand progrès dans ce domaine. L'inspection des quarante-quatre feuilles de relevés de G. Séguin représentant les peintures de Saint-Savin-sur-Gartempe et du Baptistère Saint-Jean de Poitiers, donne l'occasion de développer une longue description iconographique dans laquelle les références aux modèles antiques sont d'ailleurs encore bien vivantes ${ }^{83}$. Au gré des envois les sujets se diversifient et les nombreuses découvertes sont autant d'opportunités d'approfondir des sujets typiquement médiévaux, comme l'iconographie des saints ${ }^{84}$. Les recoupements d'informations, la diffusion des descriptions et les supports visuels permettent aux différents chercheurs de rectifier des datations, comme pour les peintures de Baume-les-Messieurs, ou même des détails iconographiques mal interprétés, soit par mauvaise exécution du relevé, soit par méconnaissance de l'auteur ${ }^{85}$. A. Didron s'interroge ainsi sur celui d'une peinture de Saint-Rémi-la-Varenne envoyé par l'architecte départemental C. Joly-Leterme ${ }^{86}$. Le dessin offre des détails incohérents certainement dûs à une mauvaise visibilité de la peinture ${ }^{87}$. Les études géographiques permettent également de distinguer des détails diffusés localement ou de grandes généralités retrouvés sur tout le territoire. É. Thévenot compare ainsi un des décors de la cathédrale de Clermont-Ferrand à des peintures « que cachaient les rayons à archives, appliqués contre le soubassement de la haute Sainte-Chapelle ${ }^{88}$. 
Les progrès dans tous les domaines de l'art médiéval ouvrent également la voie à l'iconographie comparée : ici, avec une œuvre en ivoire exposée au Louvre, là, avec une miniature illustrant un manuscrit conservé à la bibliothèque d'Angers ${ }^{89}$. Certains décors, à l'instar de celui des Anges musiciens ornant les voûtes de la chapelle axiale de la cathédrale Saint-Julien du Mans, nourrissent également les recherches dans d'autres domaines, comme celui de la musique médiévale ${ }^{90}$. Le rôle d'A. Didron, secrétaire du Comité de 1834 à 1853 dans le développement de l'étude iconographique fut, en ce sens, révélateur de la manne scientifique constituée par le rassemblement de tous les envois des correspondants ${ }^{91}$. A. Didron, fort de ces lectures, publia en son nom deux ouvrages précurseurs sur l'iconographie : les Instructions sur l'iconographie chrétienne et Iconographie chrétienne. Histoire de Dieu ${ }^{92}<$ http://gallica.bnf.fr/ark:/12148/bpt6k114544>. En 1845, il connaît un grand succès en publiant la traduction du Guide de la peinture, prétexte à une analyse comparée entre les iconographies grecque et latine devant prouver l'indépendance de l'art gothique envers l'art byzantin <http://gallica.bnf.fr/ark:/12148/ bpt6k112062s ${ }^{93}$. Dans son Iconographie chrétienne, il utilise même comme exemples ponctuels des peintures murales correspondant à certaines présentées en séance du Comité au cours des années précédentes. Il reprend ainsi les exemples des peintures murales de Montoire et de Brioude dans son chapitre sur l'iconographie du " nimbe $»^{94}$ ( fig. $\mathbf{n}^{\circ} \mathbf{6}$ ). L'ouvrage montre encore des gravures de peintures murales de Saint-Savin, tout en annonçant la publication future de l'ouvrage du Comité. Une gravure de la fresque de la Tour de Beaugency, réalisée d'après un dessin de M. A. Duchalais, montre que l'auteur a fait appel aux fonds de dessinateurs rencontrés dans le cadre de ses fonctions au Comitée ${ }^{95}$. Cet ouvrage devient ensuite une référence souvent citée pour des démonstrations d'erreurs et d'imprécisions. Ainsi M. Grille de Beuzelin, bibliothécaire à Angers fait, dans le cadre d'une recherche sur des miniatures médiévales, une rectification d'une description de mandorle attribuée à une peinture de Montoire ${ }^{96}$.

Figure 6

14. - NYMBE DIVIN $\lambda$ CHOISILLONS SURIAUSSÉS ${ }^{1}$.

Fresque du $\mathrm{xr}^{*}$ sibele, dans l'églige de Nontoire, pris de Veadome (Loir-et-Cher).

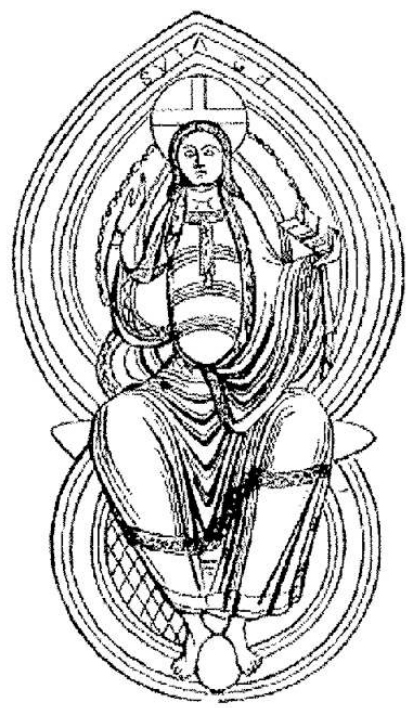

Christ en majesté. Église Saint-Gilles de Montoire, gravure illustrant l'Iconographie Chrétienne. Histoire de Dieu, publiée en 1843 par A. Didron.

(C) gallica.bnf.fr/Bibliothèque nationale de France. 


\section{Une connaissance de la technique en progression} comprendre le rôle vulgarisateur joué par le Comité ${ }^{97}$. F. Hazé, correspondant à Bourges et conservateur des monuments du Cher, s'essaie dans son rapport intitulé Observations sur la peinture à fresque, non seulement à une hiérarchisation des types de peintures murales rencontrés lors de ses fouilles, mais aussi à la description du mode d'exécution de la peinture à fresque ${ }^{98}$. L'auteur avance comme postulat de départ que ce type de peinture est supérieur à tous les autres ${ }^{99}$. Il en vend la durabilité, la rapidité d'exécution et le moindre coût ${ }^{100}$. Son texte valorise également pour la première fois la rigueur d'exécution et le talent du fresquiste, indispensables à la réalisation d'une peinture qui ne permet aucun repentir ${ }^{101}$. Sa description précise du mode d'exécution d'une fresque montre une réelle connaissance de la technique, bien qu'encore erronée pour certains détails ${ }^{102}$. Il connaît la préparation du support par la superposition des couches d'arricio et d'intonaco, et décrit le phénomène de la carbonatation. F. Hazé a également compris les enjeux d'une application rapide des couleurs et des problèmes de temps de séchage sur la transformation des tonalités. L'auteur déplore ensuite l'absence de l'utilisation de cette technique depuis le $\mathrm{XV}^{\mathrm{e}}$ siècle et manifeste sa connaissance des grands chantiers de rénovation de décors d'édifices religieux parisiens, dans lesquels sont utilisées de nouvelles techniques de peinture murale, telles que l'encaustique ou la peinture avec un liant à l'huile. Il encourage enfin également la création d'une école de formation pour la technique de la fresque et conclut son rapport par la présentation de relevés des peintures de Bois-Sir-Amé (voir fig. 3). Cette première description du mode d'exécution de la peinture à fresque manifeste l'intérêt grandissant pour les techniques de la peinture murale et la nécessité de la maîtriser pour les futurs travaux de restauration ${ }^{103}$. Alors que ces deux premières décennies d'envoi de rapports et de prospections dévoilent progressivement la richesse décorative des intérieurs des édifices français, le rôle fondamental du Comité prend tout son sens lorsqu'il s'agit de diffuser les connaissances sur la peinture murale. Son rôle en matière de mise en place d'une politique de préservation et de restauration est, quant à lui, limité et concurrencé par la Commission des Monuments historiques.

\section{Agir pour conserver les peintures murales originales : un rôle limité et concurrencé}

\section{Une intervention limitée : un rôle de veille et de relais}

Outre sa doctrine prônant l'intervention minimale sur les décors peints, le Comité a peu agi directement sur le terrain. Les informations rassemblées par le Comité sur les actions menées en Avignon, et notamment sur la prise de calques exécutée sur la peinture de S. Martini au tympan de Notre-Dame-des-Doms, sont même l'occasion de rappeler le danger des interventions directes sur les décors, puisque «des cloches se sont formées à la surface, sur l'enduit, et la pression de la main est une cause immédiate de destruction » et de favoriser la prise de relevé ${ }^{104}$. Son implication dans ce domaine se manifeste en réalité par les moyens de persuasion qu'il déploie auprès des autorités compétentes. Ses interventions auprès des ministres, préfets et tout autre acteur public ou ecclésiastique, 
permettent en effet de mobiliser le pouvoir décisionnaire qui peut bloquer les interventions irréversibles. C'est notamment le cas des peintures de l'église de SaintGenoux de Selles-Saint-Denis, vouées à la destruction en raison de la démolition programmée de l'édifice qui les conserve ${ }^{105}$ au profit d'un projet d'urbanisme. Les archives présentent pas à pas la procédure et les nombreux contacts que le Comité a dû prendre pour enrayer ce projet. Dans la majorité des affaires de conservation que le Comité traite, le rôle de la Commission des Monuments historiques sous la tutelle du ministre de l'Intérieur est prédominant.

\section{La restauration des peintures murales, une chasse gardée de la Commission des Monuments historiques?}

21 Lorsqu'il est officiellement créé, le Comité comble une faille du pouvoir central en matière de valorisation du patrimoine. S'il a l'avantage de l'âge, il voit, dès 1837, ses prétentions pour mener une politique de conservation physique des monuments balayées par la Commission des Monuments historiques spécifiquement créée à cette fin ${ }^{106}$. Or, dès son origine le Comité s'implique dans la lutte contre les restaurations destructrices et les incompréhensions des bâtiments. Dès la séance du 29 mai 1836, avant même la création de la Commission des Monuments historiques, le ministre de l'Instruction publique se plaint du zèle du Comitén ${ }^{107}$. Il voudrait que le Comité ne se cantonne qu'à publier les manuscrits et un débat sur les frontières du texte de loi se fait alors entendre. Les membres du Comité défendent leur position de pouvoir publier et agir sur les monuments ${ }^{108}$. Trouvant dans le Comité une oreille à la hauteur de leurs requêtes, les correspondants profitent en effet de l'envoi régulier de leurs rapports scientifiques pour faire remonter vers le pouvoir central les problèmes de conservation auxquels ils sont confrontés sur les chantiers. La confusion des tâches entre les deux organismes est telle que le président du Comité, le Comte de Gasparin, se doit de rappeler les fonctions de chacun des deux organismes ${ }^{109}$. Elle s'explique également par la présence de correspondants et de membres nommés dans les deux organismes ${ }^{110}$. Le rôle limité du Comité est également visible dans le domaine de la conservation de la peinture murale : il se manifeste par la transmission d'informations sur les peintures en danger à la Commission des Monuments historiques ${ }^{111}$. L'affaire des fresques de Charlieu précédemment citée est significative de l'importance de son rôle d'intermédiaire dans le sauvetage réussi d'un certain nombre de fragments de peintures murales conservés encore de nos jours au musée national du Moyen Âge ${ }^{112}$. Le Comité dénonce également, entre autres, le projet scandaleux de démolition du Palais Mazarin ${ }^{113}$. Il soutient enfin des dossiers de demandes d'aides financières auprès du ministère de l'Intérieur et obtient gain de cause, comme pour les peintures de Notre-Dame de Calais ou d'Aire-sur-la-Lys ${ }^{114}$. Si le Comité fait preuve d'une autorité réelle dans le domaine scientifique, il apparaît clairement qu'il n'a qu'un pouvoir moral d'intervention sur la conservation des peintures. Son intérêt précoce pour le décor peint mural et la primauté de son rôle de valorisation par le biais de la diffusion imprimée sont cependant progressivement remis en cause par la politique encore balbutiante mais déterminée, en ces premières années d'existence, en matière de conservation, de la Commission des Monuments historiques: cette mise en concurrence est notamment visible dans la politique de production et d'exploitation de relevés des peintures murales. 


\section{La politique d'exécution des relevés : d'un rôle de diffusion à un rôle de conservation}

Comme il a été démontré précédemment, l'implication du Comité dans la valorisation de la peinture murale n'aurait pu avoir lieu sans l'intérêt porté par un certain nombre de correspondants : les nombreux envois de dessins et le projet précoce de L. Vitet de créer une collection de dessins offrant un large répertoire d'illustrations pour les futures publications ont largement contribué à encourager l'exécution des relevés. Ainsi, à côté de l'envoi spontané de croquis et dessins purement documentaires, apparaissent d'autres dessins plus détaillés destinés, dès leur exécution, à être publiés. Cette politique d'exécution de relevés prend une toute autre tournure au moment de la préfiguration de la Notice des peintures de Saint-Savin sur Gartempe. Non seulement parce que les relevés sont, pour la première fois, commandés et financés par le Comité et qu'un dessinateur spécifique est désigné, mais aussi parce que la Commission des Monuments historiques en est l'initiatrice ${ }^{115}$. Cette entrée en matière dans la politique de relevés n'aurait sans doute pas été aussi rapide sans l'intervention décisive de P. Mérimée. Fort de son cumul de fonctions au sein des deux institutions, il a compris l'atout majeur que constituait le relevé, non seulement dans la diffusion de la connaissance du décor peint, mais aussi dans une politique de conservation des peintures en cours d'élaboration: le relevé est nécessaire pour garder le témoignage d'un patrimoine en danger que l'on ne sait pas encore restaurer efficacement. Aucun fonds de la Commission n'étant destiné à de telles affectations, on fait tout naturellement appel au Comité pour commander ces relevés ${ }^{116}$. Le travail de G. Séguin est donc soumis à l'avis des deux instances: d'abord à la Commission des Monuments historiques qui vérifie son exactitude par rapport à l'œuvre originale puis au Comité pour vérifier sa compatibilité avec un travail de publication ${ }^{117}$. Une procédure conjointe aux deux institutions se met donc progressivement en place dans la décennie 1840-1850, dans laquelle P. Mérimée joue désormais un rôle pivot. Un tournant décisif est pris dans les années 1845-1846, période pendant laquelle le Comité perd définitivement la main sur le monopole de l'exploitation des relevés. Il s'agit de l'année où la Commission des Monuments historiques semble vouloir officialiser sa politique de relevés de peintures murales, comme moyen de préservation. Elle vient en effet d'engager un dessinateur pour relever les peintures les plus insignes qui sont considérées dans un état critique: A. Denuelle, qui a déjà fait ses preuves et convaincu Mérimée de son talent lors d'un séjour en Poitou en 1840, pendant lequel il a relevé des dessins à Saint-Savin ${ }^{118}$. Mérimée prend ainsi l'initiative de présenter en séance du Comité les relevés qui lui ont été commandés. Les premiers dessins relevés à des fins conservatoires, de peintures murales de l'Hôtel Jacques Cœur à Bourges (fig. $\mathbf{n}^{\circ} 7$ ), des cathédrales d'Autun et d'Auxerre, sont ainsi présentés. Mérimée annonce aussi la politique offensive de relevés l'année suivante, pendant laquelle est programmée une vaste campagne en Avignon menée par E. Viollet-le-Duc et A. Denuelle < http:// www.culture.gouv.fr/public/mistral/mdp_fr?

ACTION=RETOUR\&USRNAME=nobody\&USRPWD $=4 \$ 4 \mathrm{P}>{ }^{119}$. En 1851 , c'est encore le vaste travail d'A. Dauvergne réalisé à la demande de la Commission des Monuments historiques sur les peintures de Saint-Michel-L'Aiguilhe qui est instruit en séance ${ }^{120}$. Le dessein de Mérimée est clair : si maintenant le ministère de l'Intérieur assure les frais de l'exécution des relevés ${ }^{121}$, il souhaite que son homologue de l'Instruction publique prenne à sa charge leurs publications. Comme il vient de le pratiquer pour l'ouvrage sur les peintures 
murales de Saint-Savin-sur-Gartempe, il souhaite établir une étroite collaboration entre le ministère de l'Intérieur et le ministère de l'Instruction publique. Progressivement le rôle de chacun entre en complémentarité et la méthode conservatoire est approuvée des deux côtés. Le rôle de Mérimée devient prépondérant, et la prise de relevé des peintures murales comme méthode de conservation est complètement adoptée par la Commission des Monuments historiques. L'envoi de dessins de peintures de l'église de Saint-Aignan près de Sedan devient ainsi le coup d'envoi d'une opération conjointe entre les deux organismes. Le Comité relaie l'information sur les peintures à ses confrères de l'Intérieur et reconnaît pleinement le rôle de la Commission des Monuments historiques qui se lance dans une vaste campagne de débadigeonnage contrôlée par ses soins, et dans une prise de relevés ${ }^{122}$.

Figure 7

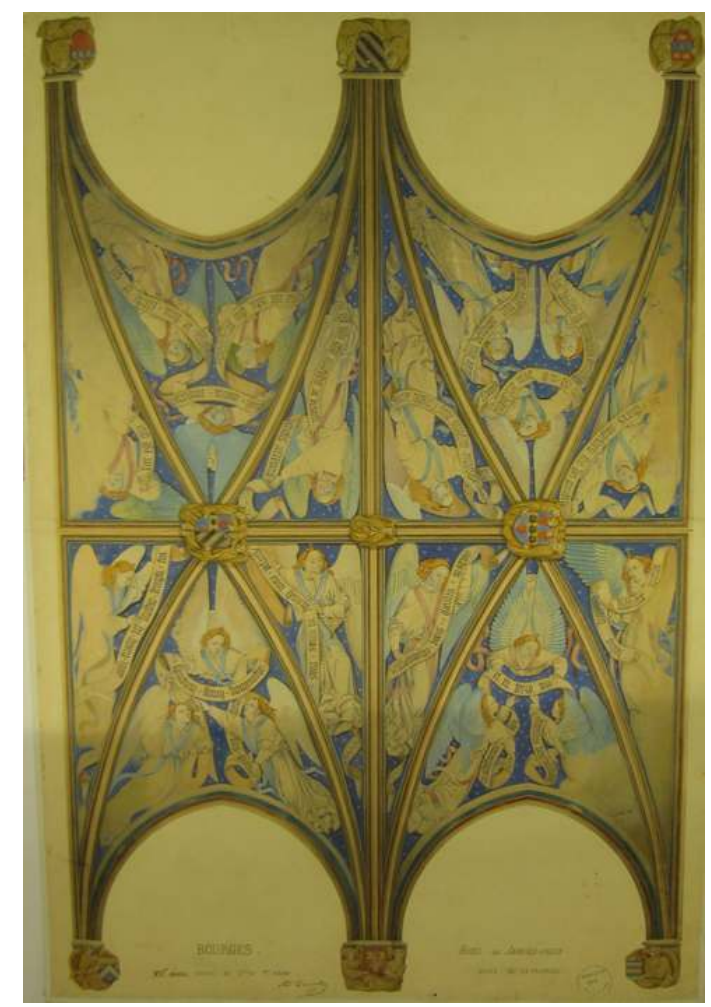

Anges portant des phylactères, décor de la voûte de la chapelle du Palais Jacques Cœur. Relevé de Denuelle, 1845, $n^{\circ} 03914$.

Phot. Médiathèque de l'architecture et du patrimoine, Élodie Jeannest, 2012. @ Ministère de la Culture, Médiathèque de l'architecture et du patrimoine.

\section{Conclusion}

Le Comité des Arts et Monuments a joué un rôle précurseur dans la valorisation de la peinture murale en France dans les années 1835-1852. Il est en effet le premier organisme d'État à avoir institué une politique d'exploitation et de diffusion durable des informations qui lui étaient rapportées des quatre coins de France sur ce sujet encore bien méconnu. Ainsi la lourde réforme qui réorganise le Comité en 1852 renforçant le contrôle de l'Empereur, restreignant les effectifs et mutualisant des séances moins nombreuses avec les deux autres sections créées, n’a que peu ébranlé le mécanisme bien 
rôdé mis en place : après cette date, les archives témoignent encore de nombreux envois de rapports et de relevés. Le Bulletin des Comités historiques continue de divulguer publiquement des découvertes et des scandales et les publications régionales réalisées par des correspondants ne cessent de croître. Il partage cependant désormais son rôle de "découvreur » de ce patrimoine fragile avec la Commission des Monuments historiques, qui a su tirer avantage de ce que le Comité avait eu la grande idée d'encourager: la collection de relevés qui avait une fonction essentielle dans la publication d'ouvrages sur la peinture murale trouve, sous la mainmise de la Commission des Monuments historiques, de nouvelles finalités. Elle devient à la fois un moyen de substitution à une restauration directe qui n'est pas encore assumée à cette époque, faute de connaissances suffisantes, et à la fois un moyen judicieux de garder la mémoire de ces décors que l'on croyait voués inévitablement à la destruction. On ne peut cependant enlever au Comité des Arts et Monuments la préséance d'avoir doté le relevé d'un rôle déterminant dans la vulgarisation de la peinture murale en France, et de lui avoir garanti, de ce fait, un long avenir dans les pratiques de valorisation du patrimoine ${ }^{123}$.

\section{BIBLIOGRAPHIE}

SOURCES :

- Archives nationales (AN)

$-\mathrm{F} 17 / 2810$

$-F 17 / 2862$

$-F 17 / 13268$

$-\mathrm{F} 17 / 13269$

$-\mathrm{F} 19 / 7823$

- Médiathèque de l'architecture et du patrimoine (MAPA)

- MAPA : Dossier Charlieu : 81/42/05

- MAPA Archives du Comité historique des Arts et Monuments, copie sur le Journal Général de l'Instruction publique : 80/02/09

- MAPA : Dossier Langon : 81/11/36

- MAPA : Dossier Baptistère de Poitiers 81/86/25

- Relevés de peintures murales, consultables sur la base Médiathek : http://www.culture.gouv.fr/ public/mistral $/ \mathrm{mdp}_{\text {fr? }}$ ACTION=RETOUR\&USRNAME=nobody\&USRPWD $=4 \$ 4 P$

\section{SOURCES IMPRIMÉES}

- Bulletin archéologique, publié par le Comité historique des Arts et monuments, vol 1 à 4, de 1843 à 1848. Paris : imprimerie administrative de Paul Dupont.

- Bulletin du Comité historique des arts et monuments, 1849-1852. 
- Prosper Mérimée. Correspondance générale, établie et annotée par PATURIER, Maurice, JOSSERAND, Pierre et MALLION, Jean. Toulouse, 1941-1967.

- BERCÉ, Françoise. Les premiers travaux de la Commission des Monuments historiques, 1837-1847. Procès-Verbaux et relevés d'architecte. Paris, 1979.

PÉRIODIQUES

- GRILLE DE BEUZELIN, Ernest. « Note sur l'église de Saint-Savin, adressée à M. de Caumont ». Bulletin Monumental, T. 1, 1834, p. 78-79.

- CASSIN DE LA LOGE, René. « Notes sur quinze aquarelles de J.-J. Jorand, reproduisant les fresques de la chapelle Saint-Gilles de Montoire (Loir-et-Cher) et conservées au musée de peinture d'Orléans ». Bulletin de la Société archéologique et historique de l'Orléanais, t. 21, 1931, p. 230.

- DAVY, Christian. « La connaissance de la peinture murale romane de la vallée du Loir un siècle après la synthèse de Henri Lafillée ». Bulletin de la Société archéologique du Vendômois, 1997, p. 35 à 41.

- BOUQUILLARD, Jocelyn. « Mérimée et la sauvegarde des fresques de Saint-Savin : une lettre inédite de Prosper Mérimée au comte Auguste de Bastard ». Études d'Histoire de l'art offertes à Jacques Thirion: Des premiers temps chrétiens au XXe siècle, publication de l'École des Chartes, $\mathrm{n}^{\circ} 3$, « Matériaux pour l'histoire », Paris, 2001.

- JEANNEST, Élodie. « Les relevés de peintures murales de Saint-Savin-sur-Gartempe aux XIX ${ }^{\mathrm{e}}$ et XX ${ }^{\mathrm{e}}$ siècles : l'aquarelle au service de la conservation ». Bulletin Monumental, tome 169-2, 2011, p. 115 à 126.

OUVRAGES

- MÉRIMÉE, Prosper. Notes de Voyage dans l'Ouest de la France. Paris, 1836.

- DIDRON, Adolphe. Iconographie chrétienne. Histoire de Dieu. Paris, 1844 ; DIDRON, Adolphe. Manuel d'iconographie chrétienne grecque et latine, avec une introduction et des notes. Paris, 1845.

- MÉRIMÉE, Prosper. Notice sur les peintures murales de l'église de Saint-Savin. Paris, 1845.

- Instructions du Comité historique des arts et monuments. Architecture gallo-romaine et architecture du Moyen Age, par Mérimée, Lenoir, Leprévost et Lenormant, membres du Comité, Paris, 1857.

- CHARMES, Xavier. Le Comité des travaux historiques et scientifiques (histoire et documents). Tome 1-3, Paris, 1896.

- BAUDOIN, Paul-Albert. La Fresque : sa technique, ses applications. Paris : Librairie centrale des beaux-arts, 1914.

- MORA, Laura et Paolo, PHILIPPOT, Paul. La conservation des peintures murales. Bologne, 1977.

- VACQUET, Étienne. Charles Joly-Leterme (1805-1885), architecte angevin. Du praticien à l'artiste. Mémoire de $3^{e}$ cycle de l'École du Louvre, sous la direction de François Loyer, 5 vol., 1998.

- PARTURIER, Maurice. Introduction à la Naissance des monuments historiques. La correspondance de Prosper Mérimée avec Ludovic Vitet (1840-1848), préface de F. Bercé. Paris : éditions du CTHS, 1998.

- LEROY, Rodolphe. Le Comité des travaux historiques et scientifiques (1834-1914). Entre animation et contrôle du mouvement scientifique en France. Thèse pour le diplôme d'archiviste-paléographe de l'École des Chartes sous la direction de J.-M. Leniaud et B. Delmas, 2001.

- LENIAUD, Jean-Michel. « Adolphe Napoléon Didron ou les « Médias » au service de l'art chrétien », chapitre XXI de La révolution des signes. L'art à l'église (1830-1930). Paris, 2007. 
- AUDUC, Arlette. Quand les monuments construisaient la nation. Le service des Monuments historiques de 1830 à 1940. Travaux et documents, $\mathrm{n}^{\circ} 25$, Comité d'histoire du ministère de la Culture, Turriers, 2008.

\section{Catalogues d'exposition}

- De fresque en aquarelle. Relevés d'artistes sur la peinture murale romane. Catalogue de l'exposition présentée à l'Abbaye de Saint-Savin du 2 juillet au 10 octobre 1994, 1994.

- PRESSOUYRE, Léon (dir.). Le dévoilement de la couleur, relevés et copies de peintures murales du Moyen Age et de la Renaissance. Catalogue de l'exposition présentée à la Conciergerie du 15/12/04 au 28/02/05, Paris, 2004.

\section{NOTES}

1. - Voir De fresque en aquarelle. Relevés d'artistes sur la peinture murale romane, catalogue de l'exposition présentée à l'Abbaye de Saint-Savin du 2 juillet au 10 octobre 1994, 1994 ; PRESSOUYRE, Léon (dir). Le dévoilement de la couleur, relevés et copies de peintures murales du Moyen Age et de la Renaissance, catalogue de l'exposition présentée à la Conciergerie du 15/12/2004 au 28/02/2005, Paris, 2004 : MAYER, Janie. « Un conservatoire des peintures murales françaises : les relevés de la Médiathèque de l'architecture et du patrimoine », p. 47-62 ; JEANNEST, Élodie. «Les relevés de peintures murales de Saint-Savin-sur-Gartempe aux XIXe et XXe siècles : l'aquarelle au service de la conservation. » Bulletin Monumental, tome 169-2, 2011, p. 115 à 126.

2. - L'idée de cette étude m'est venue à l'esprit lors de recherches menées dans le cadre de la préparation de ma thèse de doctorat dirigée par J. M. Leniaud à l'École Pratique des Hautes Études et intitulée: Le Service des Monuments historiques et les relevés de décors peints médiévaux depuis 1840 .

3. - CHARMES, Xavier. Le Comité des travaux historiques et scientifiques (histoire et documents). Tome 1-3. Paris: Imprimerie nationale, 1896 et LEROY, Rodolphe. Le Comité des travaux historiques et scientifiques (1834-1914). Entre animation et contrôle du mouvement scientifique en France. Thèse pour le diplôme d'archiviste-paléographe de l'École des Chartes sous la direction de J.-M. Leniaud et B. Delmas, 2001. Je remercie vivement l'auteur de m'en avoir autorisé la consultation et faciliter l'accès.

4. - AN/F17/13268, Séance du 18 janvier 1835.

5. - BCMH vol 1, Introduction, p. 5 « De M. Vitet était sorti le sous-comité des arts, de celui-ci naquit le comité des arts et monuments...»

6. - Pour alléger les occurrences, le «Comité historique des arts et monuments " sera appelé simplement le Comité dans le corps du texte.

7. - LEROY, Rodolphe. Op cit., Chapitre IV: L'empreinte de l'Empereur: la réorganisation administrative et scientifique du Comité (1852), p. 193-238.

8. - Circulaire du 23 juillet 1834.

9. - BA vol 1, Introduction, p. 5 : « Le 18 décembre 1837, M. de Salvandy, ministre de l'Instruction publique, érigea le sous-comité des arts en comité des arts et monuments. Il lui donna pour tâche «de publier tous les documents inédits relatifs à l'histoire des arts chez les Français, de faire connaître tous les monuments d'art en France, dans tous les genres: monuments religieux, militaires et civils, de faire dessiner et graver, pour les conserver à l'avenir, les œuvres remarquables d'architecture, de peinture, de sculpture en pierre, en marbre et en bois... »

10. - BA vol 1, Rapport sur l'année 1838, p.12-13. "Le comité est le tuteur naturel de nos monuments, il doit, par une influence latérale, sinon directe, arrêter les destructions projetées, les mutilations et les restaurations ignorantes.... » 
11. - BA, vol 3, Séance du 15 janvier 1845, p. 270 : «MM. les ministres de l'intérieur, de la justice et des cultes renvoient des communications à eux adressées par M. le ministre de l'instruction (... ). Il sera fait droit aux réclamations contenues dans ces communications, et note a été prise des renseignements qu'elles renferment. »

12. - BA vol 1, Séance du 23 juin 1841, p. 264 : «M. Schmit annonce qu'il vient d'être nommé inspecteur des cathédrales et des monuments religieux qui dépendent du ministère de la justice et des cultes. (...). Il propose de se mettre en rapport fréquent avec le comité, et de le tenir au courant de tout ce qui pourrait intéresser les études qu'il dirige et les publications qu'il entreprend. »

13. - BA vol 1, Rapport sur l'année 1838, p. 15-16: «Dans des réunions nombreuses, la commission des correspondants a discuté plusieurs points importants. Le comité (...) a décidé que les titres les plus valables pour être nommé correspondant et pour rendre des services réels, étaient les connaissances archéologiques et la science du dessin tout à la fois, cette dernière avant tout. »

14. - BA vol 1, Rapport sur l'année 1838, p. 15.

15. - BA vol 2, Séance du 19 janvier 1842, p. 2: «Un membre fait observer que trente-six départements, sur quatre-vingt-six, sont encore sans correspondants, et que le comité est privé ainsi de renseignements officiels concernant la découverte, l'étude et la conservation des monuments. »

16. - AN-F17/2830-1.

17. - AN/F17/13268, Séance du 29 mars 1835.

18. - Rapport de Mérimée à F. Guizot, le 31 octobre 1835 (Parturier Maurice (éd.), Correspondance générale de Mérimée. Paris, 1941, t. 1, p. 467). Notes de voyage dans l'Ouest de la France. Paris, 1836, p. 195-201. Il est à noter la mention antérieure de ces peintures dans le Bulletin monumental de 1834, dans une Note sur l'église de Saint-Savin, adressée à A. de Caumont par E. Grille de Beuzelin, p. 78-79.

19. - AN/F17/13268, Séance du 31 janvier 1838 : « M. Mérimée appelle de nouveau l'attention sur des fresques qui ornent encore plusieurs édifices de France. Tous les jours ces fresques se détériorent, il serait urgent de les faire dessiner surtout celles de Saint-Savin dans le département de la Vienne et du temple de Saint-Jean de Poitiers. » Voir JEANNEST, É. «Les relevés de peintures murales de Saint-Savin-sur-Gartempe aux XIXe et XXe siècles : l'aquarelle au service de la conservation. » Bulletin Monumental, tome 169-2, 2011, p. 115 à 126.

20. - BOUQUILLARD, Jocelyn. " Mérimée et la sauvegarde des fresques de Saint-Savin : une lettre inédite de Prosper Mérimée au comte Auguste de Bastard ». Dans ERLANDE-BRANDENBURG, A. et LENIAUD, Jean-Michel (dir.). Études d'Histoire de l'art offertes à Jacques Thirion: des premiers temps chrétiens au XXe siècle. («Matériaux pour l'histoire publiés par l'École nationale des Chartes », 3), Paris, 2001, p. 221-250.

21. - AN/F17/13268, Séance du 29 septembre 1838 et BCMH vol.1 Rapport sur l'année 1839, p. 89-90. Cette première affaire concernant des peintures en péril ornant un édifice appartenant au ministère de la Guerre est d'ailleurs suivie d'un certain nombre d'autres, notamment le Couvent des Jacobins de Toulouse, AN/F17/13268, Séance du 9 décembre 1850 et AN/F17/13269, Séance du 10 février 1851.

22. - BA, vol 3, Séance du 28 février 1844, p. 86 : « M. L'abbé Texier(...) donne la description des peintures murales qui ornent la crypte de la cathédrale de Limoges, dont il a envoyé au comité un fac-similé colorié, (...) M. Texier donne en outre l'indication sommaire des anciennes peintures murales qu'il a étudiées ou découvertes dans ses excursions. " Il cite notamment les décors des édifices suivants:l'église de Solignac, l'église de Bonlieu (Creuse), l'église d'Eymoutiers, la chapelle du Château du Puy-du-Val, l'église de Doraton, l'église de Saint-Pierre de Fursac (Creuse), l'église d'Aubasine (Corrèze), le château de Mazeau (commune de Peyrat-la-Nonière), l'église de la Borne (Creuse), les châteaux des Salles, Lavauguyon et de Rochechouart.

23. - BA vol 3, Séance du 26 février 1845, p. 343. 
24. - BA vol 1, Séance du 6 décembre 1840, p. 10: «Il (S. Boissérée) se propose de faire des communications au comité. À cette lettre sont jointes des réflexions sur les peintures à fresque et les anciennes peintures sur bois qui existent en France » ou AN/F17/13269, Séance du 23 novembre 1840.

25. - BA vol 3, Séance du 24 janvier 1844, p. 35 : «J'ai l'honneur de vous envoyer, (...) un exemplaire de la Description d'une salle de l'ancien Palais des rois d'Angleterre à Westminster, dite la Salle peinte, à cause des peintures remarquables, aujourd'hui entièrement détruites exécutées au treizième siècle pour servir de décoration intérieure à la salle en question. "

26. - BA vol 3, Séance du 29 mai 1844, 196 et AN/F17/13268, Séance du $1^{\text {er }}$ juin $1842:$ : M. Ch Langlois architecte à Rennes, correspondant, adresse un second rapport sur la chapelle SainteAgathe de Langon (Ille et Vilaine) et un dessin de la peinture qui décore sans doute, dès le principe, la voûte de l'abside. (...). Malheureusement la moitié de cette peinture est complètement détruite et l'on ne voit plus que le haut du corps de la déesse. Cette peinture, qui est probablement antique et présumée romaine était couverte d'une autre peinture romane qu'il a fallu détruire. La peinture de Saint-Honorat d'Arles est moins ancienne encore que celle de cette chapelle de Langon. (...) » BA vol 4, Séance du 2 mai 1846, p. 112 : « M. Mérimée annonce qu'il existait à Villars, près de St Révérien, des peintures à fresque ornant des substructions romaines : on y voyait la chasse au crocodile et des représentations de nègres. "

27. - BA vol 3, Séance du 26 février 1845, p. 343 : «M. le comte de Montalembert annonce qu'il existe au château de Thoisy la Berchère, près Saulieu (Côte d'Or), des peintures murales de l'époque de la Renaissance, exécutées dans le style de Primatice, et du plus gracieux caractère. »

28. - BA vol 3, Séance du 29 janvier 1845, p. 275.

29. - AN/F17/13269, Séance du 23 novembre 1840:«M. Jénicot (...) annonce que comme conseiller de la fabrique Saint-Jacques de Liège, il consacre ses soins à prévenir la ruine de l'église... l'on va s'occuper de réparer l'intérieur. Cette partie du monument, originairement peinte, a été couverte de plusieurs couches de lait de chaux. Il faudrait enlever le badigeon et faire reparaitre les couleurs primitives. "

30. - BA vol 3, Séance du 26 février 1845, p. 343 : «M. Charles Texier annonce qu'il vient d'être informé qu'on avait badigeonné tout récemment les belles peintures qui couvrent les murs et les colonnes de la Nativité à Bethléem. », et Séance du 19 novembre 1852, AN/F17/13269.

31. - BA vol 3, Séance du 14 février 1844, p. 78-79 : «Je termine en cet instant le huitième volume de l'Histoire du Languedoc (...) Deux fresques assez grandes, celle qui représente Charles VI fondant l'ordre de l'Espérance, et une Passion, vont être placées dans l'une des nouvelles salles de notre Musée. L'une provient du cloître des Grands Carmes; elle n'est malheureusement complète, l'autre vient du cloître des Cordeliers » et BCMH vol 1, Séance du 6 décembre 1840, p. 9 : «M. Francisque Mandet, correspondant au Puy, adresse les deux premiers numéros d'une publication encore inédite sur les monuments historiques de la Haute-Loire (...). M. Mandet fait une description détaillée de la Danse des morts qui est peinte sur la clôture du chœur, dans l'église de la Chaise Dieu; il s'élève contre un badigeonnage qui a couvert une fresque où était peinte dans l'église Saint-Michel, l'entrée du roi Charles VII au Puy. »

32. - BA vol 2, Séance du 15 avril 1842, p. 268 et AN/F17/13269, Séance du 18 novembre 1850.

33. - BA, vol 1, Séance du 23 novembre 1840, p. 75 : « M. Melchior Boisserée (...) désirerait que le comité réunît dans des ouvrages de gravure ou de lithographie d'un format convenable les monuments de la peinture sur bois, sur mur ou sur verre qui existent en France (...), on contribuerait d'une manière efficace non seulement à leur conservation, mais encore on comblerait cette grande lacune qui se trouve dans l'histoire de la peinture du Moyen Age en France. (...). Il faudrait les dessiner et pour ainsi dire, les rendre lisibles. »

34. - AN/F17/13269, Séance du 18 novembre 1850.

35. - Peu de ces relevés de cette première période de production ont été retrouvés. Voir l'hypothèse énoncée plus bas au sujet de leur absence. 
36. - Voir DAVY, Christian. «La connaissance de la peinture murale romane de la vallée du Loir un siècle après la synthèse de Henri Laffillée ». Bulletin de la Société archéologique du Vendômois, 1997, p. 35 à 41.

37. - AN/F17/13268, Séance du 9 mai 1838: A. de Bastard, A. de Laborde, P. Mérimée, A. du Sommerard et I. Taylor.

38. - AN/F17/13269, Séance de janvier 1842 : « Le président de la Séance nomme une commission qui devra s'occuper de la question du débadigeonnage. »

39. - BA vol 1, Séance du 27 janvier 1841, p. 194 : « M. Launay annonce qu'il s'est rendu à l'église de Saint-Gilles, à Montoire pour examiner une fresque représentant la Trinité et sur laquelle le comité désirait avoir des renseignements. (....) il engage cet antiquaire à joindre des dessins à ses notices, comme par le passé. Le dessin fait voir ce que le texte ne peut qu'indiquer. »

40. - AN/F17/13268, Séances du 19 juin et du 10 juillet 1837.

41. - BA vol 1, Introduction, p. 12.

42. - Quatre volumes sont ainsi publiés entre 1843 et 1848 pour les années 1838-1848. Le périodique change ensuite de titre et devient le Bulletin du Comité historique des arts et monuments. Voir LEROY, R., op cit, p. 146-148 et p. 193-194. L'auteur y voit les prémisses d'un renforcement du contrôle de l'État avant la réforme imposée par L.N. Bonaparte en 1852.

43. - BA vol 3, Séance du 24 avril 1844, p. 163.

44. - AN/F17/13268, Séance du 27 avril 1842.

45. - BA vol 2, Séance du 26 janvier 1842, p. 61 : «L'institut catholique de Lyon adresse le premier numéro d'une Revue (...) La même revue appelle l'attention du comité sur des peintures du douzième siècle et sur une chaire romane qui décore l'ancien réfectoire de l'abbaye de Charlieu, dans le Forez. (...) Des religieuses auxquelles appartiennent ces précieux débris, vont les vendre avec le réfectoire, et l'on craint que le futur acquéreur ne les conserve pas. Le comité prie $\mathrm{M}$. le ministre de faire savoir à son collègue de l'intérieur le danger que peuvent courir les peintures... » et MAPA 81/42/05.

46. - BA vol 1, Séance du 28 avril 1841, p. 302.

47. - BA vol 1, Séance du mercredi 14 avril 1841, p. 277.

48. - BA vol 1, Séance du 8 juin 1842 : «Tandis qu'on poursuit avec activité la Monographie de la cathédrale de Chartres, écrit M. Lassus, l'administration locale, ecclésiastique ou civile (je n'ai pu savoir laquelle) permet l'accomplissement d'actes de vandalisme qui ne seraient pas tolérés même dans une église de village. Au moment où le ministre de l'Instruction fait dessiner et graver et décrire avec le plus grand scrupule chaque partie de ce monument de Chartres, on voit des badigeonneurs, (...) couvrir et empâter les moulures et toute l'ornementation sculptée des chapiteaux.

49. - BA vol 3, Séance du 8 juin 1842, p. 263 : «Les habitants d'Aire sont d'une propreté excessive et préféreraient un mur bien blanc à ces peintures intéressantes, mais qui sont délabrées. (...). Le comité espère qu'on ne badigeonnera pas ces peintures, on ne les a pas découvertes pour les cacher de nouveau. (...). Le comité prie M. le ministre de renvoyer la lettre de M. Boileau à M. le ministre de l'intérieur. (...) Le secrétaire annonce que M. le ministre de l'intérieur doit allouer une somme de 500F pour nettoyer complètement ces peintures » et cathédrale du Mans : AN/ F17/13268, Séance du 27 avril 1842.

50. - AN/F17/13269, Séance du 23 novembre 1840: «Relativement au moyen d'enlever le badigeon sans altérer les peintures, M. Mérimée annonce qu'à Pise, un italien connaît ce moyen et l'a employé avec succès au Campo-Santo. M. Lenormand ajoute que ce procédé paraît défectueux et que $M$. Perrot, peintre, résidant à Pise, est persuadé qu'en France on possède un meilleur procédé. M. Perrot est à la recherche de ce moyen et se propose de l'employer pour faire reparaitre les peintures qui couvrent la belle église de Saint-Jean sur l'Arno à l'entrée de Pise. » 51. - AN/F17/13269, Séance du 23 novembre 1840 et BA vol 1, Séance du 28 avril 1841, p. 293. 
52. - AN/F17/13269, Séance du 31 mai 1843 : «M. Hazé prouve qu'il serait facile, convenable et peu coûteux de peindre aujourd'hui à fresque les monuments religieux. Le comité regrette de ne pouvoir insérer textuellement dans le Bulletin Archéologique le mémoire de M. Hazé. (...) Du reste ce document qui est renvoyé à la commission chargée d'examiner les questions qui se rattachent à la construction, à l'ornementation et à l'ameublement des nouvelles églises. »

53. - BA vol 4, Séance du 2 mai 1846, p. 113.

54. - BA vol 4, Séance du 2 mai 1846, p. 113.

55. - BA vol 4, Séance du 2 mai 1846, p. 114 : « M. Didron ajoute qu'il n'y a pas d'inconvénient à peindre sur mur les monuments modernes, mais il y aurait un grave danger à décorer de peintures murales les édifices anciens, les églises du Moyen Age. Dans des peintures de ce genre, il faut considérer l'art et la science archéologique : si l'art peut produire de belles œuvres, la science n'est pas suffisamment faite encore. Personne, en ce moment n'est capable de peindre convenablement une ancienne église. L'étude ardue et complexe de l'iconographie commence à peine. » Voir LENIAUD, Jean-Michel. « Adolphe Napoléon Didron ou les « Médias » au service de l'art chrétien », chapitre XXI. La révolution des signes. L'art à l'église (1830-1930), p. 303-330, p. 321.

56. - BA vol 4, Séance du 2 mai 1846, p. 119: «M. le président fait observer que si un grand artiste produit une œuvre en commettant des fautes d'archéologie, cette œuvre pourra être bonne, mais elle n'en serait que meilleure si les fautes n'existaient pas. La science guide le génie et ne l'entrave pas. (...) M. Didron ajoute que le Comité est institué pour étudier l'art du Moyen Age, et non pour réglementer l'art contemporain ou futur. "

57. - BA vol 4, Séance du 2 mai 1846, p. 113 et Séance du 30 mai 1846, p. 128 : « Notre Société académique a fait copier les curieuses peintures murales qui décoraient les tribunes. (...) Les copies des autres peintures ayant été exécutées avec promptitude, sont à l'aquarelle, et à cet égard, elles ne sont pas susceptibles d'être conservées longtemps, il serait donc vivement à désirer que, pour en perpétuer le souvenir, on les reproduisit avec une consciencieuse fidélité aux parois des murs où on les voyait autrefois. (...) Tous ces dessins ont été exécutés sous les yeux et avec le plus grand soin; on pourrait donc compter sur leur exactitude pour la reproduction de détails, costumes. "

58. - BA vol 4, Séance du 2 mai 1846, p. 113 : «M. le Comte de Montalembert demande des renseignements à $\mathrm{M}$. de la Cour sur le projet qu'on aurait de peindre la cathédrale du Puy. (...) Si jamais on devait peindre cette cathédrale, on prendrait pour modèles les anciennes peintures retrouvées dans la cathédrale même. »

59. - AN/F19/7823 dossier sur la cathédrale Notre Dame du Puy, rapport de A Mallay dressé le 11 février 1846 adressé au préfet de la Haute Loire : «La décoration intérieure de la cathédrale du Puy est toute tracée ; des peintures murales à fresque couvraient la voûte et les parois de l'apside, la grande coupole et les transepts; il en restait de nombreux fragments qui ont été relevés avec soin. Cette décoration devra être reproduite dans le même style, le même caractère, les mêmes sujets d'un dessin plus correct mais aussi naïf (...). »

60. - BA vol 4, Séance du 24 janvier 1846, p. 18.

61. - BA vol 2, Séance du 13 avril 1842, p. 224 et suivantes : un long chapitre, intitulé «Traits de vandalisme recueillis par le comte de Montalembert " est dévolu à la dénonciation d'actes de dégradations menés contre le patrimoine, et notamment des actes de badigeonnage.

62. - BA vol 1, Séance du14 avril 1841, p. 275 : «Didron ajoute (...) en règle générale, il faut laisser les monuments dans l'état où ils sont, et se contenter de les consolider ou de les empêcher de tomber. Une restauration quelque intelligente qu'elle soit, est rarement utile à un édifice : pour une restauration bien faite, il y en a 100 de déplorables. »

63. - BA vol 3, Séance du 24 janvier 1844, p. 50 : «M. Jénicot, avocat à Liège, correspondant, demande des avis sur la restitution des peintures qui décoraient autrefois le sanctuaire et le chœur de Saint-Jacques de Liège. Même réponse que pour Notre-Dame de Tournai: il faut 
conserver respectueusement toutes les traces des peintures anciennes que l'on peut trouver, mais il ne convient ni de rajeunir, ni de les compléter. »

64. - Parmi les différents titres, le volume sur l'architecture Gallo-romaine et l'architecture gothique publié en 1857 et regroupant les études de Mérimée, Lenoir, Lenormand et Leprévost, s'attache à donner des directives pour la peinture murale, dans son chapitre III : "Examen détaillé de l'intérieur ".

65. - LEROY, Rodolphe. Op cit, p. 144-145.

66. - AN/F17/13269, Séance du 13 janvier 1838 : «M. de Bastard annonce qu'il fait relever celles de Saint-Jean et de Saint-Savin, mais avec un grand luxe et un soin extrême car il fait calquer les dessins, ensuite réduire le calque et colorier (...) M. Mérimée croit qu'il faut une publication complète de ces peintures, trait et couleur et cependant une publication accessible à beaucoup. Il demande si M. de Bastard voudrait communiquer au comité pour en faire usage les dessins des fresques qu'il a fait relever...» Non seulement les relevés n'ont pas été retrouvés dans les archives, mais ils n'ont finalement pas servi à illustrer la publication envisagée.

67. - BOUQUILLARD, Jocelyn. Op cit, p. 221-250.

68. - BA, vol 1. Rapport de l'année 1839, p.55: «Relativement aux peintures monumentales aujourd'hui si rares en France, relativement aux vitraux, le comité a rédigé des instructions et adressé des réclamations. (...) Il aurait vivement désiré, sur la proposition de M. Mérimée, faire dessiner les nombreuses, anciennes et belles figures peintes à fresque qui décorent l'église et la crypte de Saint-Savin près de Poitiers... »

69. - AN/F17/13269, Séance du 23 juin 1841 : «M. Vitet annonce que l'église de St-Savin, près de Poitiers possède des fresques uniques en France (...). Mais ces fresques tombent en ruine et le délabrement de l'église ajoute aux autres causes de destruction, en deux ans deux grands tableaux ont disparu. Le ministre de l'Intérieur vient d'allouer une somme de $10000 \mathrm{~F}$ pour échafauder l'église et refaire le toit. Ce travail abritera les fresques et retardera leur destruction. Mais cette destruction est inévitable... »

70. - Les nombreuses discussions en Séances se rapportent aux problèmes financiers liés aux nouvelles techniques de fabrication de planches illustrées en couleurs et le choix iconographique. Voir les Séances : BA vol 1, Séance du 27 janvier 1841. 198 ; Lettre du 13 juin 1839 du ministre de l'Instruction publique au président du Comité des Arts et Monuments, citée par M. PARTURIER. Op cit; note 10, t. 2, lettre 500, p. 240-242, et AN/F17/13269, Séance du 17 janvier 1841, Séance du 27 janvier 1841, Séance du 27 avril 1842, Séance du 15 janvier 1845, BA vol 3. p. 264-265, BA vol 4, Séance du 24 janvier 1846, p. 15. Pour avoir le détail de la genèse de cet ouvrage et notamment de l'exécution de ses planches illustrées, voir JEANNEST, É., op cit.

71. - BA vol 1, Séance du 27 janvier 1841, p. 198 : Un pareil travail, entièrement nouveau, puisqu'on a jamais publié de fresques, et qui comprendrait, à lui seul, le dixième des fresques qui existaient autrefois en France, est destiné à un grand succès. »

72. - BA vol 1, Séance du 23 1840, p. 182... « En passant à Poitiers, M. Séguin a dessiné les restes de peinture qui se voient encore dans le temple de Saint-Jean et qui représentent un Christ dans une auréole en quatre feuilles et des personnages debout, drapés à la romaine, et qui paraissent être des apôtres. » et AN/F17/13269, Séance du 27 avril 1842 : «M. Mérimée annonce que les dessins de M. G. Seguin sont au nombre de 44. Mais qu'on pourrait en retrancher quatre qui sont relatifs au temple de Saint-Jean à Poitiers. "

73. - BA vol 2, Séance du 15 avril 1842, p. 218.

74. - BA vol 2, Séance du 13 avril 1842, p. 217-218: «M. de la Saussaye ajoute que M. Jorand a dessiné autrefois avec le plus grand soin toutes les peintures de la chapelle de Montoire. (...) Le comité prie M. le ministre de demander à $\mathrm{M}$. Jorand communication des dessins qu'il a exécuté d'après les fresques de Montoire. Il charge la commission des fresques de Saint Savin de choisir dans les peintures de Montoire celles qui pourraient être publiées. » 
75. - BA vol 3, Séance du 24 janvier 1844, p. 51 : «Le comité pense que les peintures qui ornent la crypte de la cathédrale de Limoges, et dont l'abbé Texier, correspondant vient d'adresser le facsimilé, pourraient faire partie de la publication des fresques de Saint-Savin. »

76. - AN/F17/13269, Séance du 12 juin 1844 : «M. Schmit propose de publier à la suite des fresques de Saint-Savin, les émaux représentant les apôtres qui décorent aujourd'hui la chapelle de la Vierge dans Saint-Pierre de Chartres. (...) M. le président nomme une commission... »

77. - BA vol 2, Séance du 10 mai 1843, p. 637 : «M. Thomas Wright annonce en outre que l'on a débadigeonné depuis quelques temps en Angleterre, les murs de beaucoup d'églises, sur ces murs on trouve presque toujours des peintures intéressantes. (...) M. Didron ajoute qu'il en est de même en France. "

78. - BA vol 4, Séance du 2 mai 1846, p. 99 : «Mérimée s'est renseigné sur le prix que coûterait une publication des peintures murales de France, exécutées en chromolithographie (...) Note est prise de ces chiffres (13000F) pour le cas ou le Ministre déciderait qu'une publication de ce genre serait faite par le Comité. »

79. - Bulletin archéologique de l'Orléanais, t. 1848-1853, Séance du 22 mars 1850, p. 75-76: « ...les peintures à fresque de la chapelle de Saint-Gilles, de Montoire, dont la Société a voté la publication dans sa Séance du 25 janvier, ont été dessinées en 1841 par M. Jorand. (...) La Société frappée de l'importance des peintures reproduites dans les cartons de M. Jorand et de la perfection avec laquelle ces cartons sont exécutés, décide qu'ils seront lithographiés à ses frais... » et CASSIN DE LA LOGE, René. « Notes sur quinze aquarelles de J.-B. Jorand, reproduisant les fresques de la chapelle Saint-Gilles de Montoire (Loir-et-Cher) et conservées au musée de peinture d'Orléans. Société archéologique et historique de l'Orléanais, n 21, 1931, p. 230.

80. - BA vol 1, Séance du 23 novembre 1840, p. 110 : «Les restes modiques de peintures à fresque qui se trouvent sur les voûtes et les murs des anciennes chapelles du palais, les parties du moins qui sont encore assez conservées, devraient naturellement entrer dans la publication. Et c'est à cause de la détérioration à laquelle avant tout ces peintures, mais plus ou moins aussi les différentes parties de l'édifice, sont exposées, que les amis de l'antiquité sont intéressés à ce que les dessins soient levés le plus tôt possible. »

81. - BA vol 3, Séance du 14 février 1844, p. 54 : «M. Martin, peintre, soumet au Comité une suite de dessins exécutés avec un grand soin d'après les bas-reliefs de l'église Notre Dame de Paris, et destinés à faire partie de la statistique monumentale de Paris, que publie le Comité (...) Il y a joint des études coloriées de la peinture qui rehaussait les fonds ou les vêtements. (...) » et BA vol 4, Séance du 30 mai 1846, p. 124: «Le secrétaire soumet au Comité deux dessins de M. Léon Gaucherel qui feront partie de la $3^{\mathrm{e}}$ livraison de la Monographie de la cathédrale de chartres. L'un sera exécuté en chromolithographie, il représente une peinture de la crypte, l'autre sera gravé... »

82. - BA vol 1, Séance du 14 avril 1841, p. 272 : «Le comité remarque avec intérêt que la société pour la conservation des monuments historiques dont le Bulletin monumental compose les Annales et le Recueil des mémoires, ne s'occupe plus exclusivement des constructions, mais commence à étudier l'iconographie qui est destinée à jeter de grandes lumières sur l'art du Moyen Age ».

83. - AN/F17/13269, Séance du 17 janvier 1841.

84. - BA vol 4, Séance du 2 mai 1846, p. 111-112 : description de saint Christophe.

85. - BA vol 4. Séance du 2 mai 1846, p. 112 : «M. Désiré Monnier, correspondant à Lons-leSaulnier, annonce que les peintures découvertes dans l'église abbatiale de Baume-les-Messieurs datent du quinzième siècle et non du douzième comme on l'avait cru. »

86. - VACQUET, E. Charles Joly-Leterme (1805-1885), architecte angevin. Du praticien à l'artiste. Mémoire de $3^{\text {e }}$ cycle de l'École du Louvre, sous la direction de François Loyer, 5 vol., 1998.

87. - BA vol 2, Séance du 31 mai 1843, « M. Didron fait observer qu'il serait nécessaire d'avoir de nouveaux renseignements, des détails circonstanciés sur cette peinture (...) Saint Jean qui devrait avoir les pieds nus est représenté ici les pieds chaussés. La coiffure de Longin est plutôt une 
couronne qu'un casque et Longin n'était ni roi, ni prince. La religion chrétienne qui tient toujours un calice, porte ici un ciboire fermé d'un couvercle. Au dessus de la tête du juif qui offre du vinaigre au C. on lit Stepiton et ce juif s'appelait stéphaton... »

88. - BA vol 1, Séance du 14 avril 1841, p. 277.

89. - BA vol 1, Séance du 27 janvier 1841, p. 194.

90. - AN/F17/13269, Séance du 22 février 1843 : « Elles représentent des anges musiciens dont les uns tiennent des instruments divers et les autres des banderoles sur lesquelles on voit de la musique notée... on la chante toujours à l'abbaye de Solesmes, seul endroit de France où l'on célèbre encore selon l'antique liturgie catholique et française... »

91. - LENIAUD, Jean-Michel, op cit, p. 307-308.

92. - DIDRON, Adolphe. Instructions sur l'iconographie chrétienne, 1841 et Iconographie chrétienne. Histoire de Dieu., Paris : Imprimerie royale, 1844.

93. - DIDRON, Adolphe. Manuel d'iconographie chrétienne grecque et latine avec introduction et notes. Paris : imprimerie royale, 1845.

94. - DIDRON, Adolphe. Instructions sur l'iconographie chrétienne, op cit, p. 23, p. 44, et p. 65.

95. - Didron a eu l'occasion de prendre connaissance de leurs différents travaux: M. Duchalais a alerté le Comité du sort des peintures à fresque de la Chapelle Saint-Genoux de Selles-Saint-Denis (Séance d'avril 1842 AN/F17/13269).

96. - BA vol 3, Séance du 10 avril 1844, p. 133 : «(...). Cette gloire est du genre de celle qui est cité dans l'Iconographie chrétienne, p. 23, comme étant une des fresques de l'église de Montoire près Vendôme. Elle ne lui est pourtant pas semblable en tout, car le nimbe, chez nous, n'a pas la croix surhaussée comme le votre. Mais je vous prie de faire attention à une chose ; dans le numéro $1^{\text {er }}$ du $9^{\text {e }}$ tome du Bulletin monumental, est inséré un rapport de M. Launay sur les fresques de la chapelle Saint Gilles à Montoire (...). Il y en a un semblable sur un des vitraux de Bourges si bien dessinés et décrits dans la grande publication de MM. Arthur Martin et Charles Cahier. Dans la seconde planche du rapport de M. Launay, le Christ a le nimbe uni, sans croisillons, et vous y verrez la preuve de l'ignorance du peintre... »

97. - AN/F17/2810 : Observation sur la peinture à fresque, par Hazé, le $1^{\mathrm{er}}$ septembre 1842.

98. - Je remercie vivement Monsieur Christian Morin, conservateur-restaurateur de peintures et peintures murales à Bergerac et Madame Françoise Auger-Feige, conservateur-restaurateur de peintures à Semur-en-Auxois des précieuses informations qu'ils ont bien voulu m'apporter sur les techniques à la lecture de ce rapport.

99. - L'auteur a raison en ce sens que la peinture à fresque est la peinture murale la plus durable, grâce au processus chimique de "calcification » qui permet le durcissement de la couche de couleur à la surface de l'enduit.

100. - Coût minimum qu'il nuance par la suite en indiquant l'importance du travail préliminaire et du salaire de l'artiste. Il se trompe pourtant en pensant qu'il est indispensable d'établir des cartons préparatoires à échelle.

101. - Une fois la couleur posée sur l'enduit frais, il est en effet quasiment impossible d'effectuer une correction, sauf à attendre que l'enduit soit sec pour revenir ensuite « à sec ».

102. - Par exemple l'auteur est persuadé de la nécessité d'appliquer une chaux hydraulique. Or le caractère hydraulique de la chaux est, au contraire, le plus possible écarté au profit d'une chaux aérienne. Pour plus de détail sur la technique de la fresque, voir : BAUDOUIN, Paul-Albert. La Fresque: sa technique, ses applications. Paris, 1914 et MORA, Laura et Paolo, PHILIPPOT, Paul. La conservation des peintures murales. Bologne, 1977.

103. - CENNINI, Cennino. Le livre de l'art ou Traité de la peinture, traduit par Victor Mottez, 1858, p. 6. Il faut noter également que le Guide de la Peinture traduit et publié par Didron en 1845 mentionne dans son premier chapitre les modes d'exécution de la technique de la fresque.

104. - AN/F17/13268, Séance 26 mai 1841 : « M. Chaix, d'Avignon, annonce que l'on calque en ce moment les peintures du porche de Notre-Dame-des Doms (...); et les calques qu'on en fait sont 
destinés à être reproduits sur verre et placés dans les fenêtres de la cathédrale d'Avignon. M. Chaix pense qu'il vaudrait mieux dessiner que calquer ces peintures délicates. »

105. - AN/F17/13269, Séance du 9 février 1842, p. 68 à 70.

106. - AUDUC, Arlette. Quand les monuments construisaient la nation. Le service des Monuments historiques de 1830 à 1940. Travaux et documents, $n^{\circ}$ 25, Comité d'histoire du ministère de la Culture, Turriers, 2008.

107. - AN/F17/13268, Séance du 29 mai 1836 : «M. le ministre croit que le comité a dépassé ses attributions, qu'il s'est trop occupé de restaurations, ce qui ressort du ministère de l'Intérieur, et pas assez de publication des documents inédits...»

108. - AN/F17/13268, Séance du 29 mai 1836 : «M. Hugo fait observer qu'il ne suffit pas de publier les monuments bâtis, mais qu'il faut encore les conserver, et léguer l'édifice lui-même plutôt que la description. (...) M. le ministre partage l'opinion de M. Hugo sur les restaurations modernes, mais il fait observer que les attributions des ministères sont distinctes : ces travaux et dépenses ressortent du ministère de l'Intérieur. »

109. - BA vol 1, Séance 12 mai 1841, p. 313-314; «Le Comité historique des arts et monuments siégeant au ministère de l'Instruction publique est entièrement distinct de la Commission des monuments historiques établie au ministère de l'intérieur. Le comité s'occupe de la recherche, de l'étude et de la description des monuments historiques; la commission est chargée de la conservation et de la restauration des mêmes monuments. Le comité renvoie à la commission les demandes de secours qui lui sont adressées, et qui concernent la conservation et la restauration des monuments. »

110. - LEROY, Rodolphe. Op cit, p. 134, « les membres partagés » représentent pour chacune des deux institutions un peu moins de $40 \%$ de leur effectif et qu'à la création de la Commission des Monuments historiques en 1837, $50 \%$ de son personnel siégeait également au Comité des arts et monuments, contre $25 \%$ dans le sens inverse. Parmi les plus célèbres et les plus actifs, citons Vitet, Mérimée, Leprévost, le Baron Taylor, Lenormant ou encore de Laborde.

111. - Le transfert des informations entre les deux organes est efficace grâce à la présence des membres doubles évoqués précédemment.

112. - Voir Supra, note 45.

113. - BA vol 3, Séance du 12 février 1845, p. 336-337 : «M. le comte de Montalembert demande que le Comité proteste contre la démolition de la Bibliothèque actuelle. (...) M. le baron Taylor ajoute qu'on s'est beaucoup occupé, au ministère de l'Intérieur, de ce projet de déplacement. On ne l'a pas approuvé et l'on a demandé instamment dans le cas où l'on détruirait la Bibliothèque Royale, que la galerie Mazarine, décorée des belles peintures de Romanelli, fut conservée (...).»

114. - BA vol.1, Séance du 23 novembre 1840, p. 82: «Les peintures de Notre-Dame de Calais : le comité remercie M. Pigault de Beaupré de ces renseignements; il prie M. le ministre de l'instruction publique de renvoyer cette lettre à $M$. le ministre de l'intérieur avec recommandation spéciale. Le débadigeonnage de Notre-Dame de Calais, confié au zèle et à l'intelligence de la commission archéologique de cette ville, mérite d'être encouragé par le ministère avec les fonds destinés à la conservation des monuments. »

115. - Pour plus de détails, voir JEANNEST, É., Op cit.

116. - BERCÉ, Françoise. Les premiers travaux de la Commission des Monuments historiques, 1837-1847. Procès-Verbaux et relevés d'architecte. Paris, 1979, Lettre du 22 juin 1840, p. 83-84 et AN/F17/13269, Séance du 17 janvier 1841.

117. - Pour une analyse critique des relevés, voir JEANNEST, É., Op cit. p. 120-121.

118. - A. Denuelle a été appelé par Mérimée lors de l'affaire des peintures murales de Charlieu. Son style plaît suffisamment à l'inspecteur des Monuments historiques pour qu'il intègre également quelques-uns de ses relevés de Saint-Savin dans sa Notice. En 1845-1846, les relevés signés de sa main et conservés par la Commission des Monuments historiques se multiplient. Voir la base Mediathek de la Médiathèque de l'Architecture et du Patrimoine: http:// 
www.culture.gouv.fr/public/mistral/mdp_fr?

ACTION=RETOUR\&USRNAME=nobody\&USRPWD=4\%24\%2534P (certaines dates antérieures à 1844, notamment celle du relevé de Vézelay ou du Baptistère de Poitiers sont erronées).

119. - BA vol 4, Séance du 21 mars 1846, p. 66 : «m. Mérimée soumet au comité des dessins de peintures murales qui se voient à bourges dans la chapelle de l'hôtel Jacques Cœur, à Autun dans une chapelle de la cathédrale, à Auxerre dans la crypte de la cathédrale. Les peintures ont été relevées par M. Denuelle, aux frais du ministère de l'intérieur. L'année prochaine MM. E Viollet le Duc et Denuelle relèveront celles du château des Papes et de Notre-Dame-des-Doms à Avignon. M. Mérimée désirerait que le comité demandât à M. le ministre de l'Instruction publique les moyens de publier ces peintures. Les peintures murales du Moyen Age exposées à de nombreuses chances de ruine sont très rares en France, quelques-unes comme celles de Charlieu viennent de disparaître en grande partie. Il serait nécessaire de les dessiner et de les publier. M. le ministre de l'Intérieur met les dessins à la disposition du Comité qui n'aurait plus qu'à faire que les frais de la publication ».

120. - AN/F17/13269, Séance du 14 avril 1851 : « Il annonce que M. Anatole Dauvergne a dessiné avec un soin extrême des peintures qui couvraient les murs de l'église Saint-Michel du Puy ... »

121. - La prise de fonction d'un dessinateur spécifique au sein de la Commission des Monuments historiques manifeste sa volonté de ne plus être soumis aux visions individuelles de chacun des correspondants dans la représentation de la peinture murale. Il marque par là les prémisses d'une standardisation du relevé. »

122. - AN/F17/13269, Séance du 14 avril 1851: «Ces peintures ont été débadigeonnées avec le plus grand soin par M. le curé de Saint-Aignan et dessinées avec non moins de soin par l'instituteur de la commune (...) il prie M. Mérimée présent à la Séance de prendre des renseignements sur les peintures (...) M. le ministre de l'intérieur pourrait sans doute accorder un secours sur le crédit affecté aux Monuments Historiques pour débadigeonner complètement ces peintures et les faire relever en dessin. M. Mérimée promet de s'occuper de cette affaire. »

123. - Voir l'article récemment publié de VALLET, Jean-Marc, DE LUCA, Livio et FEILLOU, Marie. «Une nouvelle approche spatio-temporelle et analytique pour la conservation des peintures murales sur le long terme ». In Situ [En ligne], n¹9, 2012, mis en ligne le 24 septembre 2012, consulté le 3 février 2013. URL : http://insitu.revues.org/9829.

\section{RÉSUMÉS}

Le Comité des Arts et Monuments institué par F. Guizot en 1835 a joué un rôle primordial dans la valorisation de la peinture murale en France, domaine de recherches encore balbutiant dans ce premier tiers d'un XIX siècle archéologique, romantique et polychrome. Il reçoit de ses correspondants de nombreux documents sur les peintures murales qui sont découvertes. Leurs présentations en séances sont l'occasion de débats passionnés, souvent vifs, parfois contestataires sur les problèmes de restaurations, de méthodes de débadigeonnage, de polémiques autour des nouveaux décors ou de confusions iconographiques. Le Bulletin, quant à lui, devient la tribune qui dénonce, témoigne et préconise. Le comité, héraut des causes perdues auprès des autres acteurs du pouvoir central, se voit cependant concurrencé progressivement par la Commission des Monuments historiques. 
The Comité des Arts et Monuments established by F. Guizot in 1835 played an essential role in the valuation of the wall-painting in France, domain of researches still stammering in the first third party of this archaeological, romantic and polychromatic XIXth century. It receives from its correspondents plenty of documents on the wall paintings which are discovered. Their presentations in sessions are the occasion of heated, often lively, sometimes anti-authority debates on the problems of restoration, methods of wall-cleaning, debates around the new decorations or around iconographic confusions. The Bulletin archéologique, as for it, becomes the forum which denounces, testifies and recommends. The committee, herald of lost causes with the other actors of the Central power, has gradually competition from the Commission des Monuments historiques.

\section{INDEX}

Mots-clés : Académie des Beaux-arts, Académie des Sciences, Comité des Travaux historiques et scientifiques, Comité des Arts et Monuments, Commission des Monuments historiques, conservation, débadigeonnage, Ministère de l'Instruction publique, Ministère de l'Intérieur, Ministère de la Justice et des Cultes, notice sur les peintures murales de l'église de Saint-Savin, Bulletin Archéologique, peinture murale, relevé, Société archéologique et historique de l'Orléanais

\section{AUTEUR}

\section{ÉLODIE JEANNEST}

doctorante en Histoire du Patrimoine, EPHE, sous la direction de Jean-Michel Leniaud elodie.jeannest@hotmail.fr 